\title{
Splitting methods for SPDEs: from robustness to financial engineering, optimal control and nonlinear filtering
}

\author{
Christian Bayer and Harald Oberhauser
}

\begin{abstract}
In this survey chapter we give an overview of recent applications of the splitting method to stochastic (partial) differential equations, that is, differential equations that evolve under the influence of noise. We discuss weak and strong approximations schemes. The applications range from the management of risk, financial engineering, optimal control and nonlinear filtering to the viscosity theory of nonlinear SPDEs.
\end{abstract}

\section{Introduction}

The theory of (ordinary/partial) differential equations has been very successful in modelling quantities that evolve over time. Many of these quantities can be profoundly affected by stochastic fluctuations, noise, and randomness. The theory of stochastic differential equations aims for a qualitative and quantitative understanding of the effects of such stochastic perturbations. This requires insights from pure mathematics and to deal with them in practice requires us to revisit and extend classic numerical techniques. Splitting methods turn out to be especially useful since they often allow to separate the problem into a deterministic and a stochastic part.

Christian Bayer

Weierstrass Institute, Mohrenstr. 39, 10117 Berlin, Germany e-mail: christian.bayer@wiasberlin.de

Harald Oberhauser

University of Oxford, Department of Mathematics, Andrew Wiles Building, Woodstock Road, Oxford OX2 6GG, UK e-mail: harald.oberhauser@ oxford-man.ox.ac.uk 


\section{White noise and Brownian motion}

The arguably simplest case of such a stochastic perturbation is an ODE driven by a vector field $V$ that is affected by noise. Let us model this perturbation by a sequence of random variables $N=\left(N_{t}\right)_{t \geq 0}$ which are picked up by a vector field $W$,

$$
\frac{d y_{t}}{d t}=V\left(y_{t}\right) \underbrace{+W\left(y_{t}\right) N_{t}}_{\text {Noise }} .
$$

Often a reasonable assumption is that $N=\left(N_{t}\right)_{t \geq 0}$ is white noise, that is

1. (independence) $\forall s \neq t, N_{t}$ and $N_{s}$ are independent,

2. (stationarity) $\forall t_{1} \leq \cdots \leq t_{n}$ the law of $\left(N_{t_{1}+t}, \cdots, N_{t_{n}+t}\right)$ does not depend on $t$,

3. (centered) $\mathbb{E}\left[N_{t}\right]=0, \forall t \geq 0$.

Above properties imply that the trajectory $t \mapsto N_{t}$ cannot be continuous, and even worse if we assume that $\mathbb{E}\left[N_{t}^{2}\right]=1$ then $(\omega, t) \mapsto N_{t}(\omega)$ is not even measurable (see $[59,40])$. Putting mathematical rigour aside, let us rewrite the above differential equation as an integral equation, i.e. we work with $B_{t}=\int_{0}^{t} N_{r} d r$ and since integration smoothes out we expect $B=\left(B_{t}\right)_{t \geq 0}$ to have nicer trajectories than $N$. In this case the above becomes

$$
\mathrm{d} y_{t}=V\left(y_{t}\right) \mathrm{d} t+W\left(y_{t}\right) \mathrm{d} B_{t} \text { resp. } y_{t}=\int_{0}^{t} V\left(y_{r}\right) \mathrm{d} r+\int_{0}^{t} W\left(y_{r}\right) \mathrm{d} B_{r}
$$

It turns out that $B=\left(B_{t}\right)_{t \geq 0}$ can be rigorously defined as a stochastic process i.e. a collection of $(\omega, t)$-measurable random variables carried on some probability space $(\Omega, \mathscr{F}, \mathbb{P})$. This process $B$ is the well-known Brownian motion ${ }^{1}$.

Definition 1. We call a real-valued stochastic process $B=\left(B_{t}\right)_{t \geq 0}$ defined on a probability space $(\Omega, \mathbb{P})$ a one-dimensional Brownian motion if

1. $B_{0}=0$ and $t \mapsto B_{t}$ is continuous (a.s.),

2. $\forall t_{1}<\cdots<t_{n}$ and $n \in \mathbb{N}, B_{t_{2}}-B_{t_{1}}, \ldots, B_{t_{n}}-B_{t_{n-1}}$ are independent,

3. $\forall s, t, t-s>0, B_{t}-B_{s} \sim \mathscr{N}(0, t-s)$.

The trajectories $t \mapsto B_{t}(\omega)$ are "degenerate": they are highly oscillatory, of infinite length, (statistically) self-similar and possess a rich fractal structure; see Figure 1. Developing a theory that can deal with such trajectories is what makes stochastic calculus such a fascinating and rich subject. Finally, let us note that while Brownian motion is probably the most important stochastic process, there are many other

\footnotetext{
${ }^{1}$ Named after the botanist Robert Brown who observed in 1827 that pollen grains suspended in water execute continuous but jittery motions. The physical explanation was given by Albert Einstein in 1905 (his "annus mirabilis": small water molecules hit the pollen) and a little earlier Marian Smoluchowski had already emphasized the importance of this process for physics. Further important contributions are due to Louis Bachelier, Andrey Kolmogorov, Paul Lévy, Joseph Doob, Norbert Wiener and finally Kyoshi Ito
} 
classes of noise that appear in the real-world and are not covered by the Brownian (e.g. so-called fractional Brownian motion [55]) and many of the methods we present here are not limited to the Brownian or even semimartingale setting.
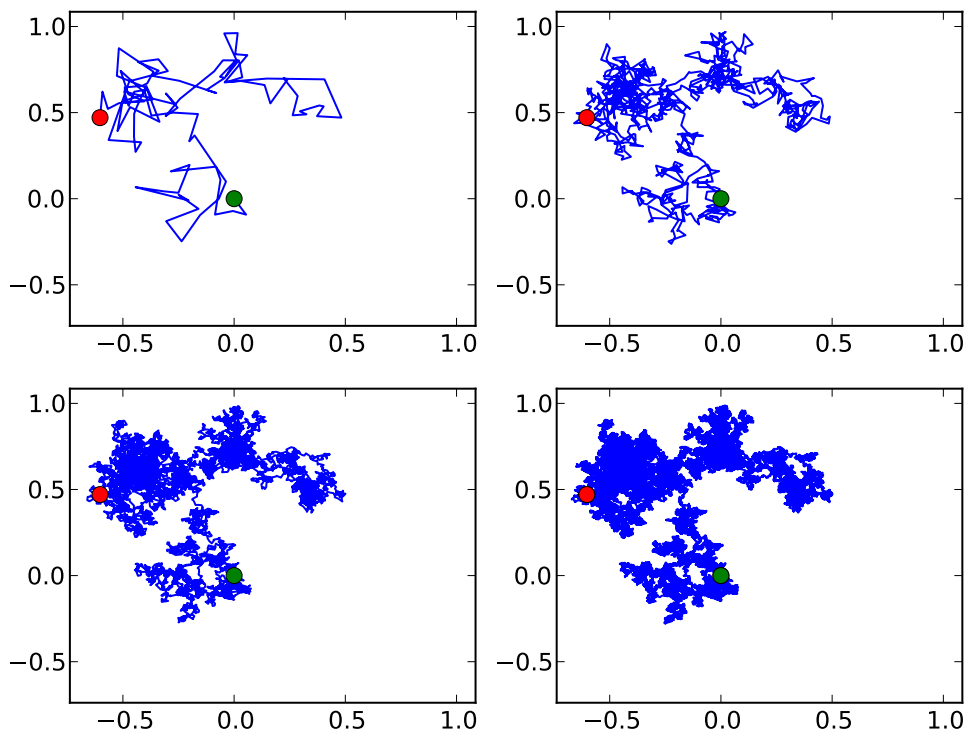

Fig. 1 The piecewise linear interpolation between the points of a two dimensional Brownian motion started at $t=0$ at $(0,0)$ (green circle), stopped at $t=1$ (red circle) and sampled at time steps of size $\left\{10^{-2}, 10^{-3}, 10^{-4}, 10^{-5}\right\}$.

\section{Stochastic integrals}

The Gaussianity of Brownian increments implies $B_{t}-B_{s} \sim N \sqrt{t-s}$ for $N \sim \mathscr{N}(0,1)$, hence we can expect at best a Hölder-modulus of $1 / 2$ and the problem of giving meaning to $\int_{0}^{t} W\left(y_{r}\right) d B_{r}$ appears. To see what goes wrong with Riemann-Stieltjes integrals consider integrating a one-dimensional Brownian trajectory against itself: with dyadic partitions of $[0,1], t_{i}^{n}=i .2^{-n}$ a direct calculation shows that

$$
\sum_{i} B_{\frac{i+1}{2}+t_{i}^{n}}\left(B_{t_{i+t}^{n}}-B_{t_{i}^{n}}\right) \text { and } \sum_{i} B_{t_{i}^{n}}\left(B_{t_{i+1}^{n}}-B_{t_{i}^{n}}\right)
$$

both converge (for a.e. Brownian trajectory $B(\omega)$ ) but to different limits. The difference of their limits equals $1 / 2$ times the $n \rightarrow \infty$ limit of 


$$
\sum_{i}\left(B_{t_{i+1}^{n}}-B_{t_{i}^{n}}\right)^{2}
$$

and the quantity (3) is the so-called quadratic variation process $\left([B]_{t}\right)_{t \geq 0}$ of the Brownian motion $B$. Kyoshi Ito developed a powerful integration theory by generalizing the above limit construction (2). He gave meaning to $\int_{0}^{t} \phi_{r} d B_{r}$ for a large class of stochastic processes $\phi$ by taking the $L^{2}(\Omega)$-limit of $\sum_{i} \phi_{t_{i}^{n}}\left(B_{t_{i+1}^{n}}-B_{t_{i}^{n}}\right)$ as $n \rightarrow \infty$. A crucial ingredient is that the integrand $\phi$ does not "look into the future evolution of $B$ "2. For many applications like mathematical finance this is a desirable property. Instead of the right sum in in (2) one can also use the left sum, i.e. the mid-points $\phi_{\left(t_{i}^{n}+t_{i+1}^{n}\right) / 2}$, to arrive at a different notion of stochastic integration called the Stratonovich integral, denoted $\int_{0}^{t} \phi_{r} \circ d B_{r}$. Above approaches to stochastic integration are not limited to Brownian motion and can be extended to the class of semimartingales. Ito and his successors (especially the "Ecole de Strasbourg") developed a complete theory that gives existence and uniqueness for stochastic equations of the form (1); see [63, 43, 60, 59].

\section{Ito's change of variable formula}

Stochastic calculus is not a first order calculus: the change of variable formula, called "Ito's Lemma", reads as

$$
f\left(t, B_{t}\right)=f\left(0, B_{0}\right)+\int_{0}^{t} \frac{\partial f}{\partial t}\left(r, B_{r}\right) d r+\int_{0}^{t} \frac{\partial f}{\partial x}\left(r, B_{r}\right) d B_{r}+\frac{1}{2} \int_{0}^{t} \frac{\partial^{2} f}{\partial x^{2}}\left(r, B_{r}\right) d[B]_{r} .
$$

A big advantage of the Stratonovich integral is that it follows a first order calculus,

$$
f\left(t, B_{t}\right)=f\left(0, B_{0}\right)+\int_{0}^{t} \frac{\partial f}{\partial t}\left(r, B_{r}\right) d r+\int_{0}^{t} \frac{\partial f}{\partial x}\left(r, B_{r}\right) \circ d B_{r} .
$$

All this is only the starting point for one of the most exciting mathematical developments of the twentieth century and to make the above rigorous requires much more care - we refer the reader to the many excellent introductory texts $[61,59,41,63]$.

\section{A drawback: discontinuity of the solution map}

While stochastic calculus had tremendous impact on theory and applications it has several shortcomings; two which are relevant for this article are that it is, firstly,

\footnotetext{
${ }^{2}$ More precisely, the relevant property of the Brownian motion here is that $B$ is a martingale. Geometrically, this is an orthogonality relation between the increments $B_{t}-B_{s}$ and the path up to time $s$. Hence, the construction works in a geometric $L^{2}(\Omega)$ sense which allows to take advantage of this structure.
} 
limited to the class of semimartingales as noise (this for example excludes fractional Brownian motion) and secondly, that a very basic object, namely the solution map associated with (1),

$$
B \mapsto Y,
$$

is not continuous in uniform norm (or any other reasonable norm). Over the last 20 years, Terry Lyons and collaborators [54, 52, 49, 32, 35] devolped a robust and completely analytic/algebraic approach to such differential equations; this is the socalled "theory of rough paths". It is not meant to replace stochastic calculus but it complements it where it runs into trouble; especially in view of splitting results this robustness becomes very useful and gives for example continuity of the solution map.

\section{Structure of this chapter}

In Section 2 we introduce the main topic of this chapter, namely that splitting schemes can be derived from robustness of the solution map. In Section 3 we recall some key results from the the theory of rough paths which give a quantitative and qualitative understanding of the regularity of this solution map.

Splitting methods for $\mathrm{S}(\mathrm{P}) \mathrm{DEs}$ are naturally divided in strong and weak schemes. The goal of strong schemes is to approximate the solution $Y$ of a S(P)DE (or a function of it, $f(Y)$ ) for a given realization of the noise. On the other hand, for many applications it is sufficient to only approximate the expected value $E[f(Y)]$. Strong approximations are discussed in Section 4 and applications to nonlinear filtering and optimal control are given in Section 5. In Sections 6 we discuss weak splitting schemes for $\mathrm{S}(\mathrm{P}) \mathrm{DEs}$ and their rate of convergence; we recall a popular weak approximation scheme called "cubature on Wiener space" and show that it has a natural interpretation as a splitting scheme. In Section 7 we present three applications of splitting schemes in financial engineering: efficient implementations for popular stochastic local volatility models [2]; a calibration of the Gatheral Double Mean Reverting model to market data [3]; and finally the Heath-Jarrow-Morton interest rate model [23].

\section{Background}

This chapter is inspired by a view on stochastic differential equations that emerged over the last 15 years, namely the theory of rough paths due to Terry Lyons and collaborators; for further developments and introduction see [54, 51, 52, 35, 32, 50, 29]). This theory complements classic Ito-calculus and provides new, if not revolutionary insights, on how differential equations react to complex input signals. One of the earliest new applications was the so-called "cubature on Wiener 
space" of Kusuoka-Lyons-Victoir [53, 46]. Bayer, Dörsek, Teichmann among others $[23,67,22,5]$ then showed that these methods can be applied to the infinitedimensional setting that is needed by SPDEs. More recently more applications were developed both in finite and in infinite dimensions (we survey some of these in Section 7). In a somewhat different direction, the work of Friz-Oberhauser [30] combined robustness from rough path theory with viscosity PDE methods to derive splitting schemes for strong approximations of (nonlinear) SPDEs.

Of course, splitting-up methods have appeared much earlier in stochastic calculus and we emphasize that these techniques remain highly relevant and form the basis of much of the recent developments that we present here. However, instead of giving a "horizontal" historical account we decided to give a "vertical" snapshot of what we believe are some exciting current developments in theory and applications. Unfortunately, this implies that we cannot do full justice to the existing rich literature. Nevertheless, we would like to point the reader to some classic articles as a starting point: one of the earliest motivations comes from the theory of nonlinear filtering and we mention pars pro toto the work of Bensoussan and Glowinski [6] and Bensoussan, Glowinski and Răşcanu [7, 8], Elliott and Glowinski [24], Florchinger and Le Gland [47, 27], Gyöngy and Krylov [36], Nagase [56], Sun and Glowinski [65] and Lototsky, Mikulevicius and Rozovskii [48]. The more general field of splitting is overwhelmingly large, so that we again cannot hope to give a balanced literature review. Some general works we want to mention are Jentzen and Kloeden [42], Debussche [20], Gyöngy and Krylov [37], Răşcanu and Tudor [62] Hausenblas [38] and, finally, Yan [68]. Let us finally stress that we consider partial differential equations driven by a temporal (possibly also spacial) noise, not partial differential equations with spacial noise, another very active research field in applied mathematics (see, for instance, Schwab and Gittelson [64]).

\section{From robustness to splitting schemes}

On an abstract level, we have to understand how the output path (the solution of a differential equation) of a complex system (a differential equation) responds to an input path (e.g. time and noise). In this section we show that if a continuous dependence between output and input signal holds, then splitting results follow immediately.

\section{A toy example}

Let us consider the simple example of a quantity $y$ whose evolution over time is described by the differential equation

$$
\frac{\mathrm{d} y_{t}}{\mathrm{~d} t}=V\left(y_{t}\right)+W\left(y_{t}\right), y_{0} \in \mathbb{R}^{e}
$$


where $V, W$ are Lipschitz continuous vector fields on $\mathbb{R}^{e}$. We identify this differential equation as a special case of the integral equation

$$
y_{t}=\int_{0}^{t} V\left(y_{r}\right) d a_{r}+\int_{0}^{t} W\left(y_{t}\right) d b_{r}, y_{0} \in \mathbb{R}^{e}
$$

where $a$ and $b$ are continuous, real-valued paths that are regular enough that above integrals have meaning. While finite 1-variation as recalled in Definition 2 is sufficient for the Riemann-Stieltjes integrals, we will treat paths having much less regularity in later parts of this chapter. Equations of type (6) are often called controlled (differential/integral) equations and $a, b$ are referred to as the controls or also as the driving paths/signals. Such equations arise naturally in the engineering sciences and have been very well studied (see the seminal work of work of Brockett, Sussmann, Fliess, et al. [11, 26, 66]). We henceforth use the shorthand/differential notation

$$
\mathrm{d} y_{t}=V\left(y_{t}\right) d a_{t}+W\left(y_{t}\right) d b_{t}, y_{0} \in \mathbb{R}^{e}
$$

to denote (6). A basic question is the regularity of the solution map

$$
(a, b) \mapsto y .
$$

Obviously, the answer depends on what norms we use to measure distances between paths. What might be somewhat surprising is that the above mapping, defined on smooth paths

$$
C^{1}\left([0, T], \mathbb{R}^{2}\right) \rightarrow C^{1}\left([0, T], \mathbb{R}^{e}\right),
$$

is not even continuous under the usual uniform norm $|a|_{\infty}=\sup _{t \in[0, T]}\left|a_{t}\right|$; we invite the reader to find an example for this discontinuity and come back to this issue in detail in Example 1. Motivated by this, we introduce a cascade of metrics that are stronger than the uniform norm.

Definition 2. Let $x$ be a continuous path defined on $[0, T]$ that takes values in a complete metric space $(E, d)$. For every $p \geq 1$ the $p$-variation norm of $x$ is defined as

$$
|x|_{p-v a r}=\sup _{\substack{n \in \mathbb{N},\left(t_{1}, \ldots, t_{n}\right): \\ 0 \leq t_{1}<\cdots<t_{n} \leq T}}\left(\sum_{i=1}^{n} d\left(x_{t_{i+1}}, x_{t_{i}}\right)^{p}\right)^{1 / p}
$$

We denote the subset of $C([0, T], E)$ of paths finite $p$-variation norm by $C^{p-v a r}([0, T], E)$.

Standard arguments show that $\left(C^{p-v a r}([0, T], E),|\cdot|_{p-v a r}\right)$ is a Banach space. We now see that the $p$-variation norm resolves the non-continuity of the uniform norm.

Theorem 1 (Robustness [32].). Let $V, W: \mathbb{R}^{e} \rightarrow \mathbb{R}^{e}$ be Lipschitz continuous and $(a, b) \in C^{1-v a r}\left([0, T], \mathbb{R}^{2}\right)$. Then there exists a unique solution $y \in C^{1-v a r}\left([0, T], \mathbb{R}^{e}\right)$ to the controlled differential equation

$$
\mathrm{d} y_{t}=V\left(y_{t}\right) \mathrm{d} a_{t}+W\left(y_{t}\right) \mathrm{d} b_{t}, y_{0} \in \mathbb{R}^{d}
$$


and the map $(a, b) \mapsto y$ is continuous in 1-variation norm $|\cdot|_{1-v a r}$.

\section{Lie and Strang splitting}

The connection with splitting is now immediate: Fix $\Delta>0$ and divide $[0, T]$ into intervals of size $\Delta>0$; further, denote $t_{\Delta}=\left\lfloor\frac{t}{\Delta}\right\rfloor \Delta, t^{\Delta}=t_{\Delta}+\Delta$ and define two time-changes (real-valued increasing paths) $\phi^{\Delta}, \varphi^{\Delta}$,

$$
\phi_{t}^{\Delta}= \begin{cases}t_{\Delta}+2\left(t-t_{\Delta}\right) & , \text { if } t \in\left[t_{\Delta}, \frac{t_{\Delta}+t^{\Delta}}{2}\right] \\ t^{\Delta} & , \text { if } t \in\left[\frac{t_{\Delta}+t^{\Delta}}{2}, t^{\Delta}\right], \varphi_{t}^{\Delta}=\phi_{t+\frac{\Delta}{2}}^{\Delta} .\end{cases}
$$

In other words, we approximate $t \mapsto(t, t)$ with $t \mapsto\left(\phi_{t}^{\Delta}, \varphi_{t}^{\Delta}\right)$ as $\Delta \rightarrow 0$, as depicted in Figure 2. Basic arguments show that this convergence holds in $p$-variation norm for every $p>1$ (for $p=1$ it is not true).
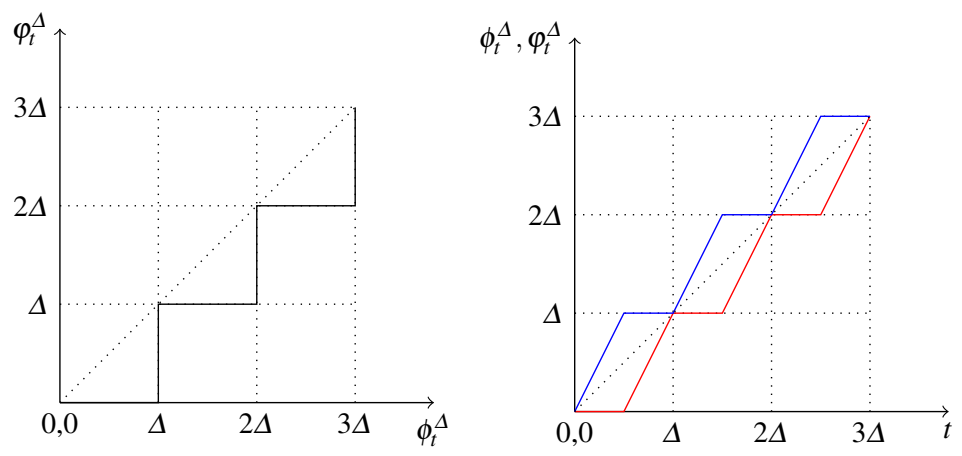

Fig. 2 (Lie-Splitting) The two-dimensional path $t \mapsto\left(\phi^{\Delta}, \varphi^{\Delta}\right)$ approximates the identity $t \mapsto(t, t)$ and exactly one of $\frac{d \phi_{t}^{\Delta}}{d t}, \frac{d \varphi_{t}^{\Delta}}{d t}$ is 0 for any given time $t \geq 0$. This gives rise to the so-called Liesplitting scheme.

This particular choice of control paths immediately implies a splitting result since by composition of $a$ (resp. $b$ ) with $\phi^{\Delta}$ (resp. $\varphi^{\Delta}$ ) we flow at any moment in time either along $V$ or along $W$. This approach is quite different from applying the Trotter-Kato formula to semigroups but it has already been used several times in the literature; see work of Le Gland [47], Gyöngy and Krylov [37]).

The above choice of $a^{\Delta}, b^{\Delta}$ yields the classic Lie-splitting but obviously other choices are possible, for example we recover the Strang-Splitting scheme by using

$$
\tilde{\phi}_{t}^{\Delta}=\phi_{t+\frac{\Delta}{4}}^{\Delta} \text { and } \tilde{\varphi}_{t}^{\Delta}=\tilde{\phi}_{t+\frac{\Delta}{2}}^{\Delta}
$$

To state it precisely, we introduce the notion of solution operators. 

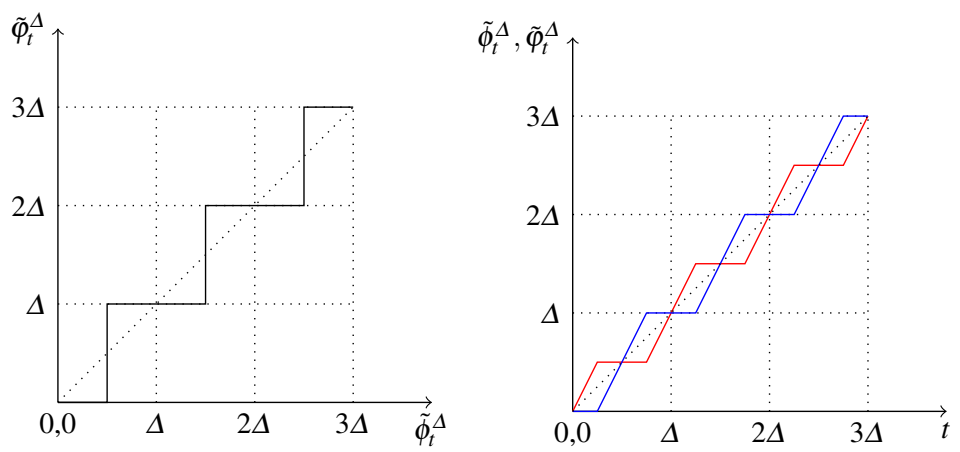

Fig. 3 (Strang-Splitting) The two-dimensional path $t \mapsto\left(\tilde{\phi}^{\Delta}, \tilde{\varphi}^{\Delta}\right)$ approximates the identity $t \mapsto$ $(t, t)$ better than $t \mapsto\left(\phi_{t}^{\Delta}, \varphi_{t}^{\Delta}\right)$ as depicted in Figure 2. Therefore it should be not surprising that Strang-splitting leads to better rates than Lie-splitting.

Definition 3. For every $y_{0} \in \mathbb{R}^{e}$ denote by $P_{t}^{\Delta, V} y_{0}$ the solution at time $t$ of the controlled differential equation

$$
\mathrm{d} y_{t}^{\Delta}=V\left(y_{t}^{\Delta}\right) \mathrm{d}\left(a \circ \phi^{\Delta}\right)_{t}
$$

started at $y_{0}^{\Delta}=y_{0}$. Similarly denote by $Q_{t}^{\Delta, W} y_{0}$ the solution at time $t$ of the controlled differential equation

$$
\mathrm{d} y_{t}^{\Delta}=W\left(y_{t}^{\Delta}\right) \mathrm{d}\left(b \circ \varphi^{\Delta}\right)_{t}
$$

started at $y_{0}^{\Delta}=y_{0}$.

Corollary 1 (Splitting). We have $\forall t>0$

$$
\lim _{\Delta \rightarrow 0}\left|\left(P_{\Delta}^{\Delta, V} Q_{\Delta}^{\Delta, W}\right)^{\lfloor t / \Delta\rfloor} y_{0}-y_{t}\right|=0
$$

where $y$ is the solution of the differential equation (6) started at time 0 with $y_{0}$. Moreover, the convergence even holds uniformly in $t$.

Proof. A simple calculation shows that the path $\left(a \circ \phi^{\Delta}, b \circ \varphi^{\Delta}\right)$ converges to the the path $t \mapsto(t, t)$ with uniform 1-variation bounds, i.e. $\sup _{\Delta>0}\left|a^{\Delta}\right|_{1-v a r}+$ $\sup _{\Delta>0}\left|b^{\Delta}\right|_{1-v a r}<\infty$. The claim then follows from a slight variation of Theorem $1 ;$ see [30].

\section{Highly oscillatory paths and the Lie brackets of vector fields}

We now replace the path $b$ in (6) by one with highly oscillatory trajectories (which is typical for many stochastic processes). 
Example 1. Consider the sequence of paths $\left(a_{t}^{n}, b_{t}^{n}\right)_{n}=\left(\frac{1}{n} \cos 2 \pi n^{2} t, \frac{1}{n} \sin 2 \pi n^{2} t\right)$ and note that it converges for the uniform norm to $\left(a^{0}, b^{0}\right)=(0,0)$ as $n \rightarrow \infty$. Define the vector fields $W\left(y^{1}, y^{2}, y^{3}\right):=\left(1,0, \frac{-y^{2}}{2}\right)^{T}$ and $V\left(y^{1}, y^{2}, y^{3}\right):=\left(0,1, \frac{y^{1}}{2}\right)^{T}$ and denote by $y^{\Delta}$ the solution of

$$
\mathrm{d} y_{t}^{\Delta}=V\left(y_{t}^{\Delta}\right) \mathrm{d} a_{t}^{\Delta}+W\left(y_{t}^{\Delta}\right) \mathrm{d} b_{t}^{\Delta} \text { with } y_{0}^{\Delta}=y_{0} \in \mathbb{R}^{3} .
$$

A simple calculation then shows that $y_{1}^{\Delta}$ does not converge as $\Delta \rightarrow 0$ to $y_{0}$ (the solution of (10) applied with $\Delta \equiv 0$ ).

In the above Example 1, the highly oscillatory motions of the driving signals affect the evolution of $y$ not directly via $V$ or $W$ but via their Lie bracket $[V, W]=V \cdot W-$ $W \cdot V$ which picks up the signed area (recall the Green/Stokes formula)

$$
(s, t) \mapsto \frac{1}{2}\left(\int_{s}^{t} a_{r}^{n} d b_{r}^{n}-\int_{s}^{t} b_{r}^{n} d a_{r}^{n}\right)
$$

swept out by $\left(a^{n}, b^{n}\right)$ during the time interval $(s, t)$. To sum up, the highly oscillatory behaviour of the driving signal leads to a subtle interplay between the iterated integrals of the driving signal and the Lie brackets of the involved vector fields that can destroy continuity of the solution map. However, was central to our derivation above of the Lie and Strang-splittings. Below we show how the theory of rough paths provides the needed continuity and gives us a very robust way to solve differential equations driven by such highly oscillatory paths.

\section{Rough path theory}

We still have to give meaning to differential equations driven by non-smooth paths and study the properties of the associated solution map. Example 1 suggests that the iterated integrals

$$
\int_{s<r_{1}<t} \mathrm{~d} x_{r_{1}}, \int_{s<r_{1}<r_{2}<t} \mathrm{~d} x_{r_{1}} \otimes \mathrm{d} x_{r_{2}}, \ldots, \int_{s<r_{1}<\cdots<r_{n}<t} \mathrm{~d} x_{r_{1}} \otimes \cdots \otimes \mathrm{d} x_{r_{n}} .
$$

of the driving signal $x$ (resp. their linear combinations) play a special role when the path is highly oscillatory. In general, these integrals will not make sense as Riemann-Stieltjes integrals if $x$ is of unbounded variation. However, the theory of rough paths shows that it is enough to find a sequence of tensors that "behaves algebraically" like such a sequence of iterated integrals to derive the well-posedness of differential equations driven by this "iterated integrals". 


\section{The space of iterated integrals}

The sequence of iterated integrals has a rich algebraic structure. Let us first give the definition for the case of a bounded variation path.

Definition 4. For every $u, v$ such that $0 \leq u \leq v \leq T$ define $\Delta_{u, v}^{1}=\{(s, t): u \leq s \leq t \leq v\}$. Let $x \in C^{1-v a r}\left([0, T], \mathbb{R}^{d}\right),(s, t) \in \Delta_{0, T}$ and $k \in \mathbb{N}$. We define the iterated integrals $\int_{\Delta_{s, t}^{k}} \mathrm{~d} x \otimes \cdots \otimes \mathrm{d} x \in\left(\mathbb{R}^{d}\right)^{\otimes k}$ recursively as

$$
\int_{\Delta_{s, t}^{1}} \mathrm{~d} x:=x(t)-x(s) \text { and } \int_{\Delta_{s, t}^{k}}^{\mathrm{d} x \otimes \cdots \otimes \mathrm{d} x}:=\int_{s \text { times }}^{t} \int_{\Delta_{s, r}^{k-1}} \underbrace{\mathrm{d} x \otimes \cdots \otimes \mathrm{d} x}_{(k-1) \text { times }} \otimes \mathrm{d} x_{r} .
$$

Recall that the space $\left(\mathbb{R}^{d}\right)^{\otimes k}$ used above is the space of $k$-tensors which has as basis $\left(e_{i_{1}} \otimes \cdots \otimes e_{j}\right)_{i, j \in\{1, \ldots, d\}} \cdot$

Definition 5. Let $x \in C^{1-v a r}\left([0, T], \mathbb{R}^{d}\right)$ and $(s, t) \in \Delta_{0, T}^{1}$. The signature of $x$ over $[s, t]$, denoted by $S(x)_{s, t}$, is the element of $\bigoplus_{k=0}^{\infty}\left(\mathbb{R}^{d}\right)^{\otimes k}$ given as

$$
S(x)_{s, t}=\left(1, \int_{\Delta_{s, t}^{1}} \mathrm{~d} x, \int_{\Delta_{s, t}^{2}} \mathrm{~d} x \otimes \mathrm{d} x, \ldots\right)
$$

with the convention that $\left(\mathbb{R}^{d}\right)^{\otimes 0}=\{1\}$. Similarly, we define for $n \in \mathbb{N}$ the truncated signature of $x$ over $[s, t]$, denoted $S^{n}(x)_{s, t}$, as the element of $\bigoplus_{k=0}^{n}\left(\mathbb{R}^{d}\right)^{\otimes k}$ given as

$$
S^{n}(x)_{s, t}=(1, \int_{\Delta_{s, t}^{1}} \mathrm{~d} x, \int_{\Delta_{s, t}^{2}} \mathrm{~d} x \otimes \mathrm{d} x, \ldots, \int_{\Delta_{s, t}^{n}} \underbrace{\mathrm{d} x \otimes \cdots \otimes \mathrm{d} x}_{n \text { times }}) .
$$

We call the path $t \mapsto S^{n}(x)_{0, t}$ the step- $n$ lift of $x$.

The above definition is not efficient concerning the state space since it does not account for the recursive structure of $S^{n}(x)$ and we can hope to work with a much smaller subspace of $\bigoplus_{k=0}^{n}\left(\mathbb{R}^{d}\right)^{\otimes k}$. With slight abuse of notation denote by $\otimes: \bigoplus_{k=0}^{n}\left(\mathbb{R}^{d}\right)^{\otimes k} \rightarrow \bigoplus_{k=0}^{n}\left(\mathbb{R}^{d}\right)^{\otimes k}$ the natural extension of the tensor multiplication to the graded space $\bigoplus_{k=0}^{n}\left(\mathbb{R}^{d}\right)^{\otimes k}$, i.e. for

$$
g=\sum_{k=0}^{n} \sum_{i_{1}, \ldots, i_{k}} g^{i_{1} \ldots i_{k}} e_{i_{1}} \otimes \cdots \otimes e_{i_{k}}, h=\sum_{k=0}^{n} \sum_{i_{1}, \ldots, i_{k}} h^{i_{1} \ldots i_{k}} e_{i_{1}} \otimes \cdots \otimes e_{i_{k}} \in \bigoplus_{k=0}^{n}\left(\mathbb{R}^{d}\right)^{\otimes k}
$$

define

$$
g \otimes h=\sum_{k=0}^{n} \sum_{l, m: l+m=k} g^{i_{1} \ldots i_{l}} h^{i_{1} \ldots i_{m}} e_{i_{1}} \otimes \cdots \otimes e_{i_{k}} \otimes e_{i_{1}} \otimes \cdots \otimes e_{i_{m}}
$$


We can now describe the algebraic structure of the subspace of $\bigoplus_{k=0}^{n}\left(\mathbb{R}^{d}\right)^{\otimes k}$ that contains the iterated integrals.

Theorem 2 ([32]). For $n \geq 1$ and $d \geq 1$ define $G_{n, d}:=\left\{S(x)_{0,1}: x \in C^{1-\operatorname{var}}\left([0, T], \mathbb{R}^{d}\right)\right\}$.

Then

1. $\left(G_{n, d}, \otimes\right)$ is a Lie group,

2. $G_{n, d}=\exp \mathfrak{g}_{n, d}$ where $\left(\mathfrak{g}_{n, d},[\cdot, \cdot]\right)$ is Lie algebra and

3. $\mathfrak{g}_{n, d}=\mathbb{R}^{d} \oplus\left[\mathbb{R}^{d}, \mathbb{R}^{d}\right] \oplus \cdots \oplus\left[\mathbb{R}^{d},\left[\mathbb{R}^{d},\left[\cdots,\left[\mathbb{R}^{d}, \mathbb{R}^{d}\right] \cdots\right]\right]\right]$

We call $G_{n, d}$ the free step-n Lie group with d generators and $\mathfrak{g}_{n, d}$ the free step-n Lie algebra with d generators. The geodesic (so-called Carnot-Caratheodory) distance $d_{C C}$ turns $\left(G_{n, d}, d_{C C}\right)$ in a metric space.

\section{(Weak) geometric rough paths}

Since $\left(G_{n, d}, d_{C C}\right)$ is a complete metric space, Definition 2 applies and we can speak of paths of bounded $p$-variation - this is exactly the definition of a weak geometric $p$-rough path.

Definition 6. Let $p \geq 1$ and $n=\lfloor p\rfloor$. We define the space of weak geometric $p$ rough paths as

$$
C^{p-v a r}\left([0, T], G_{n, d}\right):=\left\{\mathbf{x} \in C\left([0, T], G_{n, d}\right): d_{p-v a r}(0, \mathbf{x})<\infty\right\}
$$

(here 0 denotes constant path that takes the value of the neutral element of the group $\left.G_{n, d}\right)$.

Example 2 (The Brownian rough path). Let $B$ be a two-dimensional Brownian motion. This gives rise to the $G_{2,2}$-valued path

$$
\begin{aligned}
\mathbf{B}_{t} & =\underbrace{\left(1, B_{t}, \int_{0}^{t} d B \otimes d B\right)}_{\in G_{2,2}} \\
& =\exp \underbrace{\left(B_{t}^{1} e_{1}+B_{t}^{2} e_{2}+\frac{1}{2}\left(\int_{0}^{t} B_{r}^{1} d B_{r}^{2}-\int_{0}^{t} B_{r}^{2} d B_{r}^{1}\right)\left(e_{1} \otimes e_{2}-e_{2} \otimes e_{1}\right)\right)}_{\in \mathfrak{g}_{2,2}}
\end{aligned}
$$

where the integrals are understood as (Stratonovich) stochastic integrals. One can show that $\mathbf{B} \in C^{p-v a r}\left([0, T], G_{2,2}\right)$ for any $p>2$, see $[54,32]$. 


\section{Differential equations driven by rough paths}

Ito's approach to differential equations driven by highly oscillatory stochastic processes exploits the underlying probabilistic structure of the driving signal. Lyons $[54,51,50]$ developed a different approach that relies only on analytic and algebraic methods; most important for us, it comes with a cascade of metrics which provide the needed continuity of the solution map.

Theorem 3 ('Universal Limit Theorem": Existence, uniqueness and continuity of RDEs; see [54, 32]). Let $p \in(2,3), d \geq 1, \mathbf{x} \in C^{p-v a r}\left([0, T], G_{2, d}\right)$ and $V_{i} \in C_{b}^{3}\left(\mathbb{R}^{e}, \mathbb{R}^{e}\right)$. There exists a $y \in C^{p-v a r}\left([0, T], \mathbb{R}^{e}\right)$ such that for every sequence $\left(x^{n}\right)_{n} \subset C^{1-v a r}\left([0, T], \mathbb{R}^{d}\right)$ such that $d_{p-v a r}\left(S_{2}(x), \mathbf{x}\right) \rightarrow 0$, the solutions of the ODE

$$
d y_{t}^{n}=V\left(y_{t}^{n}\right) d x_{t}^{n} \equiv \sum_{i} V_{i}\left(y_{t}^{n}\right) d\left(x^{n}\right)_{t}^{i}
$$

converge uniformly to $y$. We say that $y$ is a solution of the RDE driven by $\mathbf{x}$ and write

$$
d y_{t}=V\left(y_{t}\right) d \mathbf{x}_{t} .
$$

The solution map is uniformly continuous on compact sets, that is for every $R>0$ the map

$$
\begin{aligned}
\left(y_{0}, x\right) & \mapsto y \\
\mathbb{R}^{e} \times\left\{d_{p-v a r}(x, 0)<R\right\} & \rightarrow C^{p-v a r}\left([0, T], G_{2, d}\right)
\end{aligned}
$$

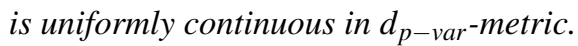

\section{Summary}

Rough path theory provides us with a machinery to solve differential equations driven by non-smooth signals (like Brownian motion, semimartingales but also many other classes of noise that are not covered by classic stochastic calculus). As opposed to the Ito-theory it not only requires the trajectory of the driving signal as input but also its "iterated integrals"; to be precise, it requires a set of tensors that "behave like" classical Riemann-Stieltjes iterated integrals. Finding efficient state spaces for these "enhanced paths" required us to work with nonlinear spaces, i.e. Lie groups. In return we get a completely analytic and algebraic approach that provides the well-posedness of such differential equations, and the rough path theory comes with a cascade of metrics which makes the solution map continuous (the metric $d_{p-v a r}$ for $p \geq 1$ ). Such a robustness is in stark contrast with Ito's theory and allows us to translate our simple splitting proof from the toy example in Section 2 to the case of $\mathrm{S}(\mathrm{P}) \mathrm{DEs}$. 


\section{Strong splitting schemes for SPDEs}

In this section we extend the splitting method to parabolic PDEs that evolve under the influence of noise. A large class of such stochastic partial differential equations (SPDEs) is of the form

$$
\left\{\begin{aligned}
\mathrm{d} u & =F\left(t, x, u, D u, D^{2} u\right) \mathrm{d} t+\sum_{i=1}^{d} \Lambda_{i}(t, x, u, D u) \mathrm{d} z_{t}^{i} & & \text { on }[0, T] \times \mathbb{R}^{n} \\
u(0, x) & =u_{0}(x) & & \text { on } \mathbb{R}^{n}
\end{aligned}\right.
$$

where $u=u(t, x)$ is scalar-valued, $F$ denotes a nonlinear, (possibly degenerate) elliptic differential operator, $\Lambda$ is affine linear in $(u, D u)$ and $z \in C\left([0, T], \mathbb{R}^{d}\right)$ is a multidimensional path with the same (or worse) regularity properties as Brownian trajectories.

Several issues appear: firstly, even if $\Lambda \equiv 0$, then the nonlinearity of $F$ implies that we cannot hope for a smooth solution $u \in C^{1,2}\left([0, T] \times \mathbb{R}^{n}, \mathbb{R}\right)$. Therefore we have to work with a suitable concept of generalized solutions. Secondly, the path $z$ is not differentiable and similar to our toy example, we have to give appropriate meaning to $\Lambda(t, x, u, D u) \circ \mathrm{d} z_{t}$. Put simply, we solve the first problem by working with the theory of viscosity solutions and the second problem with the theory of rough paths.

\section{Approximating time}

As in our toy example in Section 2, we now want to look at equation (11) as a special case of

$$
\left\{\begin{aligned}
\mathrm{d} u^{\Delta} & =F\left(t, x, u^{\Delta}, D u^{\Delta}, D^{2} u^{\Delta}\right) \mathrm{d}\left(a \circ \phi^{\Delta}\right)_{t}+\sum_{i=1}^{d} \Lambda\left(t, x, u^{\Delta}, D u^{\Delta}\right) \circ \mathrm{d}\left(z^{i} \circ \varphi^{\Delta}\right)_{t}, \\
u^{\Delta}(0, x) & =u_{0}(x)
\end{aligned}\right.
$$

However, the situation is more subtle.

Example 3. Consider $n=1, F\left(t, x, u, D u, D^{2} u\right)=D^{2} u$ and $\Lambda \equiv 0$ in which the above reduces to the one-dimensional heat equation: $\mathrm{d} u^{\Delta}=D^{2} u^{\Delta} \mathrm{d}\left(a \circ \phi^{\Delta}\right)_{t}$. Then one cannot hope for continuity of $(a, z) \mapsto u$ since this requires to give meaning to the heat equation when $\frac{\mathrm{d}\left(a \circ \phi^{\Delta}\right)_{t}}{\mathrm{~d} t}<0$, i.e. when time is run backwards which is in general not well-posed.

We simply resolve the above issue by replacing $C^{1-v a r}$ by a smaller class of paths.

Proposition 1 ([30]). Define

$$
C_{0}^{1,+}([0, T], \mathbb{R})=\left\{\xi \in C^{1}([0, T], \mathbb{R}): \xi_{T}=T, \dot{\xi}_{t}>0 \forall t\right\}
$$

and its closure $C_{0}^{1-v a r,+}([0, T], \mathbb{R}):={\overline{C_{0}^{1,+}([0, T], \mathbb{R})^{\mid}}}^{||_{\infty}}$ where $|a|_{\infty} \equiv \sup _{t \in[0, T]}\left|a_{t}\right|$. Then 


$$
\begin{aligned}
& \begin{array}{l}
C_{0}^{1-v a r,+}([0, T], \mathbb{R})=\left\{\xi \in C_{0}([0, T], \mathbb{R}): \xi_{T}=T \text { and } \exists \xi^{\text {cont }} \in L^{1}([0, T], \mathbb{R}),\right. \\
\left.\qquad \exists \xi^{\text {sing }} \in C^{1-v a r}\left([0, T], \mathbb{R}_{\geq 0}\right), \xi^{\text {sing }}=0 \text { a.s. and } \xi_{t}=\xi_{t}^{\text {sing }}+\int_{0}^{t} \xi_{r}^{\text {cont }} d r\right\}
\end{array} \\
& \text { and } C_{0}^{1-v a r,+}([0, T], \mathbb{R}) \subsetneq C_{0}^{1-v a r}([0, T], \mathbb{R}) \text {. }
\end{aligned}
$$

\section{Viscosity solutions of PDEs}

Given a map

$$
F:[0, T] \times \mathbb{R}^{n} \times \mathbb{R} \times \mathbb{R}^{n} \times \mathbb{S}^{n} \rightarrow \mathbb{R}
$$

(with $\mathbb{S}^{n}$ denoting the set of symmetric $(n \times n)$-matrices) that is proper in the sense that

$$
\begin{aligned}
F(t, x, r, p, A) & \leq F(t, x, r, A+B) \forall A \in \mathbb{S}^{n} \text { and } B \geq 0 \\
r & \mapsto F(t, x, r, A) \text { is increasing, }
\end{aligned}
$$

then the theory of viscosity solutions provides well-posedness for parabolic PDEs of the form

$$
\left\{\begin{aligned}
\partial_{t} u-F\left(t, x, u, D u, D^{2} u\right) & =0 & & \text { on }[0, T] \times \mathbb{R}^{n} \\
u(0, x) & =u_{0}(x) & & \text { on } \mathbb{R}^{n}
\end{aligned}\right.
$$

More precisely, if $u:[0, T] \times \mathbb{R}^{n} \rightarrow \mathbb{R}$ is bounded and uniformly continuous then we call $u$ a subsolution of the PDE (13) if for every $\varphi \in C^{1,2}\left([0, T] \times \mathbb{R}^{n}, \mathbb{R}\right)$ it holds that whenever $(\hat{t}, \hat{x})$ is a local maximum of

$$
(t, x) \mapsto u(t, x)-\varphi(t, x)
$$

then

$$
\partial_{t} \varphi(\hat{t}, \hat{x})-F\left(\hat{t}, \hat{x}, \varphi, D \varphi, D^{2} \varphi\right) \leq 0
$$

Similarly, we define supersolutions and call $u$ a solution if it is a sub- and supersolution. Viscosity theory provides comparison results, that is given a subsolution $v$ and a supersolution $w$ of (13) this guarantees that

$$
v \leq w
$$

(note that this immediately implies uniqueness of solutions), see [15, 25]. 


\section{Robustness for (nonlinear) SPDEs}

We can now have an educated guess of a good solution concept for the nonlinear SPDE (11): let us approximate $t \mapsto\left(t, z_{t}\right)$ by a sequence $\left(\xi^{n}, z^{n}\right)_{n} \subset C^{1}\left([0, T], \mathbb{R}^{d}\right)$ of smooth paths. Then for every fixed $n \in \mathbb{N}$ we can speak of a viscosity solution $u^{n} \in$ $\operatorname{BUC}\left([0, T] \times \mathbb{R}^{n}\right)$ - the space of real-valued, bounded and uniformly continuous functions - of

$$
\left\{\begin{aligned}
\mathrm{d} u^{n} & =F\left(t, x, u^{n}, D u^{n}, D^{2} u^{n}\right) \mathrm{d} \xi^{n}+\sum_{i=1}^{d} \Lambda\left(t, x, u^{n}, D u^{n}\right) \mathrm{d} z_{t}^{n ; i} & & \text { on }[0, T] \times \mathbb{R}^{n}, \\
u^{n}(0, x) & =u_{0}(x) & & \text { on } \mathbb{R}^{n} .
\end{aligned}\right.
$$

We expect that $\left(u^{n}\right)_{n}$ converges to a function $u \in \mathrm{BUC}\left([0, T] \times \mathbb{R}^{n}\right)$ as $n \rightarrow \infty$ and it is natural to identify this function $u$ as the solution of the SPDE (11). It turns out that it is natural to define convergence of the sequence $\left(\xi^{n}, z^{n}\right)_{n}$ to $\left(t, \mathbf{z}_{t}\right)$ if

$$
\begin{array}{r}
\sup _{n}\left\|S\left(z^{n}\right)\right\|_{p-\operatorname{var} ;[0, T]}+\sup _{n}\left|\xi^{n}\right|_{1-\text { var }}<\infty \\
d_{0}\left(z, S\left(z^{n}\right)\right)+\left|\xi_{t}^{n}-t\right|_{\infty} \rightarrow_{n} 0
\end{array}
$$

holds. Here $d_{0}(\mathbf{x}, \mathbf{y}) \equiv \sup \sum_{t_{i}} d_{C C}\left(\mathbf{x}_{t_{i}, t_{i+1}}, \mathbf{y}_{t_{i}, t_{i+1}}\right)$ where the sup is taken over all partitions $\left(t_{i}\right)$ and we use the notation $\mathbf{x}_{t_{i}, t_{i+1}} \equiv \mathbf{x}_{t_{i}}^{-1} \mathbf{x}_{t_{i+1}}$ for increments in the group. Let us take this as definition of a solution.

Definition 7. Let $z \in C_{0}^{0, p-v a r}\left([0, T], G_{[p], d}\right), \xi \in C_{0}^{1-v a r,+}([0, T], \mathbb{R})$. Let

$$
\left(z^{n}, \xi^{n}\right)_{n} \subset C_{0}^{0, p-v a r}\left([0, T], G_{[p], d}\right) \times C_{0}^{1-v a r,+}([0, T], \mathbb{R})
$$

be a sequence that converges to $\left(t, z_{t}\right)$ in the sense of (16) and assume that there exists for every $n$ a unique viscosity solution $u^{n}$ of the PDE (15). We call every accumulation point (in the metric of uniform convergence on compacts) of $\left(u^{n}\right)$ a solution of the RPDE

$$
\left\{\begin{aligned}
\mathrm{d} u & =F\left(t, x, u, D u, D^{2} u\right) \mathrm{d} \xi_{t}+\Lambda(t, x, u, D u) \circ \mathrm{d} z_{t} & & \text { on }[0, T] \times \mathbb{R}^{n} \\
u(0, x) & =u_{0}(x) & & \text { on } \mathbb{R}^{n}
\end{aligned}\right.
$$

If this limit is unique and does not depend on the choice of the approximating sequence $\left(\xi^{n}, z^{n}\right)_{n}$ and the solution map

$$
(\xi, z) \mapsto u
$$

is continuous then we say that (17) is robust in the rough path sense.

It is clear that the above robustness in rough path sense immediately gives a splitting result when use the time changes $\left(\phi^{1 / n}, \varphi^{1 / n}\right)$ from Section 2 to define the approximating sequence

$$
\left(\xi \circ \phi^{1 / n}, z \circ \varphi^{1 / n}\right)_{n}
$$


In Section 5 below we show that large classes of SPDEs are robust in rough path sense as defined above.

\section{Applications of strong schemes to nonlinear filtering and optimal control}

\section{Nonlinear filtering}

In many areas of science, the quantities of interest are not available for direct measurement. Fortunately, we can make reasonable inferences about them by combining mathematical models that describe their evolution with partial observations of these quantities. These partial observations are typically corrupted by noise and we need to account for this. Applications range from cryptography, tracking and guidance, the study of the global climate, to the management of risk in a economic context (see for example $[17,10,28,34])$. Consider a Markov process $(X, Y)$ that takes its values in $\mathbb{R}^{d_{\text {sig }}+d_{\text {obs }}}$ with its dynamics given by

$$
\begin{cases}\mathrm{d} X_{t}=\mu\left(X_{t}\right) \mathrm{d} t+\sigma\left(X_{t}\right) \mathrm{d} B_{t} & \text { (signal) } \\ \mathrm{d} Y_{t}=h\left(X_{t}\right) \mathrm{d} t+\mathrm{d} \tilde{B}_{t} & \text { (observation) }\end{cases}
$$

Here, $B$ and $\tilde{B}$ are multidimensional Brownian motions that are defined on some probability space $(\Omega, \mathscr{F}, \mathbb{P})$. The goal is to compute for a given real-valued function $f$ the conditional expectation

$$
\pi_{t} f \equiv \mathbb{E}\left[f\left(X_{t}\right) \mid \mathscr{Y}_{t}\right]
$$

i.e. to find the best estimate for $f\left(X_{t}\right)$ given the observation $\sigma$-algebra ${ }^{3} \mathscr{Y}_{t}=$ $\sigma\left(\left\{Y_{r}, r \in[0, t]\right\}\right) \vee \mathscr{N}$ with $\mathscr{N}$ denoting the $\mathbb{P}$-null-sets. From basic principles it follows that there exists a measurable map $\phi_{t}^{f}: C\left([0, t], \mathbb{R}^{d_{Y}}\right) \rightarrow \mathbb{R}$ such that

$$
\phi_{t}^{f}\left(\left.Y\right|_{[0, t]}\right)=\pi_{t} f \mathbb{P}-\text { a.s. }
$$

and our problem reduces to effectively calculate this functional $\phi_{t}^{f}$.

\section{Clark's robustness problem}

In practice, only a finite number of observations $\left(Y_{t_{i}}\right)_{i}$ of $Y$ is available and we evaluate $\phi_{t}^{f}$ along some continuous interpolation of these points, $Y^{\text {interpolated }}$. Of course we expect that

3 There are some subtle measure-theoretic issues which we gloss over but refer the reader to [1] for more details. 


$$
\phi_{t}^{f}\left(\left.Y^{\text {interpolated }}\right|_{[0, t]}\right) \simeq \phi_{t}^{f}\left(\left.Y\right|_{[0, t]}\right)
$$

but this is not guaranteed by (18), as the interpolation is a path of bounded variation, hence a null-set under the Wiener measure or any equivalent measure, see [16] for a detailed discussion. Clark [13] sketched a proof (a rigorous argument was given later by Clark and Crisan [14]) that if $B$ and $\tilde{B}$ are uncorrelated, then there exists a functional $\phi_{t}^{f \text {,robust }}$ that is continuous in supremum norm and fulfills (18). In the correlated case, such a functional cannot exist but recently (see [16]), it was shown that in the correlated case there exists also a functional $\phi_{t}^{f \text {,robust }}$ defined on the space of rough paths such that

$$
\phi_{t}^{f, \text { robust }}(Y)=\pi_{t} f \mathbb{P}-\text { a.s and } Y \mapsto \phi_{t}^{f, \text { robust }}(Y)
$$

is continuous in rough path metric. This solves Clark's robustness problem (for semimartingale piecewise linear approximations converge in the appropriate rough path metric).

\section{The Kallianpur-Striebel and Zakai equations}

Theorem 4 (Kallianpur-Striebel). There exists a probability measure $\tilde{\mathbb{P}}$ on $(\Omega, \mathscr{F})$ such that

1. $\tilde{\mathbb{P}}$ is equivalent to $\mathbb{P}$,

2. $\left.\frac{\mathrm{d} \tilde{\mathbb{P}}}{\mathrm{dP}}\right|_{\mathscr{F}}=\exp \left(-\int_{0}^{t} h\left(X_{s}\right) \cdot \mathrm{d} B_{s}-\frac{1}{2} \int_{0}^{t}\left|h\left(X_{s}\right)\right|^{2} \mathrm{~d} s\right)$,

3. the observation process $Y$ is a Brownian motion under $\tilde{\mathbb{P}}$,

4. for every $f \in B\left(\mathbb{R}^{d_{\text {sig }}}\right)$ 一the space of real-valued, bounded, measurable functions on $\mathbb{R}^{d}$-and every fixed $t>0$

$$
\pi_{t} f=\frac{\tilde{\mathbb{E}}\left[f\left(X_{t}\right) \exp \left(\int_{0}^{t} h\left(X_{s}\right) \cdot \mathrm{d} Y_{s}-\frac{1}{2} \int_{0}^{t}\left|h\left(X_{s}\right)\right|^{2} \mathrm{~d} s\right) \mid \mathscr{Y}_{t}\right]}{\tilde{\mathbb{E}}\left[\exp \left(\int_{0}^{t} h\left(X_{s}\right) \cdot \mathrm{d} Y_{s}-\frac{1}{2} \int_{0}^{t}\left|h\left(X_{s}\right)\right|^{2} \mathrm{~d} s\right)\right]} \mathbb{P} \text { and } \tilde{\mathbb{P}} \text { a.s. }
$$

Proof. This can be found in every text book on nonlinear filtering; see for example $[1,17]$.

It turns out that it is advantageous to work with an non-normalized version of the inferred probability measure $\pi$. Indeed, if we define for every $f \in B\left(\mathbb{R}^{d_{\text {sig }}}\right)$

$$
\rho_{t} f=\pi_{t} f \cdot \tilde{\mathbb{E}}\left[\exp \left(\int_{0}^{t} h\left(X_{s}\right) \cdot \mathrm{d} Y_{s}-\frac{1}{2} \int_{0}^{t}\left|h\left(X_{s}\right)\right|^{2} \mathrm{~d} s\right)\right]
$$

then obviously $\pi_{t} f=\frac{\rho_{t} f}{\rho_{t} 1}$. The Fokker-Planck/Kolmogorov forward equation is a PDE given by the generator of $X$ that describes the time evolution of the density of the diffusion $X$ via a parabolic PDE with the elliptic differential operator 


$$
A=\sum_{i, j}\left(\sigma^{T} \cdot \sigma\right)^{i, j} \frac{\partial^{2}}{\partial x_{i} \partial x_{j}}+\sum_{i} \mu^{i} \frac{\partial}{\partial x_{i}} .
$$

The Zakai equation can be seen as an extension that incorporates the additional information we get from the observation process $Y$. Indeed, set $h \equiv 0$ in Theorem below to recover the Fokker-Planck equation.

Theorem 5 (The Zakai SDE; uncorrelated case). Under standard assumptions ${ }^{4}$ we have $\tilde{\mathbb{P}}$-a.s. for every $t \geq 0$ and every $f \in B\left(\mathbb{R}^{d_{\text {sig }}}\right)$ that

$$
\left(\rho_{t} f\right)=\pi_{0} f+\int_{0}^{t} \rho_{s}(A f) \mathrm{d} s+\int_{0}^{t} \rho_{s}\left(f h^{T}\right) \mathrm{d} Y_{s} .
$$

Proof. See for example [1, Chapter 3].

The above applies to the case when $B$ and $\tilde{B}$ are uncorrelated. In the correlated case a slight variation of the above Zakai SDE holds (an extra differential operator appears in the stochastic integral against $Y$ ).

\section{Splitting for the Zakai SPDE}

It is advantageous to work with densities instead of measures. Indeed, under wellknown conditions $\rho$ has a density $u$ and we can write

$$
\rho_{t}(A)=\int_{A} u(t, x) \mathrm{d} x
$$

for some $u \in \operatorname{BUC}\left([0, T] \times \mathbb{R}^{n}\right)$. In this case we can rewrite the above (infinitedimensional) Zakai SDE from Theorem 5 for the unnormalized measure $\rho$ as a SPDE for the density $u$. Since the generator of the signal $X$ is linear, second order parabolic it is not surprising that the resulting SPDE will be linear (with linear noise). In fact, our setup is more general than needed by the nonlinear filtering application and below we treat general semi-linear PDEs (of which the SPDE for the density $u$ is a special case).

Assumption 1. Let

$$
L(t, x, r, p, M)=\operatorname{Tr}[M(x) \cdot X]+b(x) \cdot p+f(x, r)
$$

with $M(x)=\sigma(x) \sigma^{T}(x), \sigma: \mathbb{R}^{n} \rightarrow \mathbb{R}^{n \times m}$ and $b: \mathbb{R}^{e} \rightarrow \mathbb{R}^{e}$ bounded, Lipschitz in $x$. Further, let $f: \mathbb{R}^{n} \times \mathbb{R} \rightarrow \mathbb{R}$ be continuous, bounded whenever $r$ is bounded and with a lower Lipschitz bound, i.e.

$$
f(x, r)-f(x, s) \geq c(r-s) \forall r \geq s, x \in \mathbb{R}^{n} .
$$

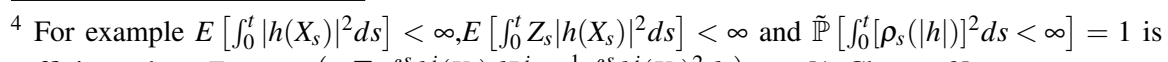
sufficient where $Z_{s}=\exp \left(-\sum_{i} \int_{0}^{s} h^{i}\left(X_{r}\right) d B_{r}^{i}-\frac{1}{2} \int_{0}^{s} h^{i}\left(X_{r}\right)^{2} d r\right)$; see [1, Chapter 3] 
Assumption 2. Let

$$
\Lambda(t, x, r, p)=p \cdot \sigma_{k}(t, x)+r \cdot v_{k}(t, x)+g_{k}(t, x)
$$

where $\sigma, v$ and $g$ are $\operatorname{Lip}^{\gamma}$ for $\gamma>p+2$.

Theorem 6 (Well-posedness of linear RPDEs). Let $z \in C^{0, p-v a r}\left([0, T], \mathbb{R}^{d}\right)$ and let $L$ and $\Lambda$ fulfill assumption (1) resp. (2). Then

$$
\left\{\begin{aligned}
\mathrm{d} u & =L\left(t, x, u, D u, D^{2} u\right) \mathrm{d} t+\sum_{i=1}^{d} \Lambda(t, x, u, D u) \circ \mathrm{d} z_{t}^{i} & & \text { on }[0, T] \times \mathbb{R}^{n}, \\
u(0, x) & =u_{0}(x) & & \text { on } \mathbb{R}^{n} .
\end{aligned}\right.
$$

is robust in rough path sense.

Proof. We only sketch the idea of the proof for the case

$$
\left\{\begin{aligned}
\mathrm{d} u & =\sigma^{2}(t, x) D^{2} u \mathrm{~d} t+\sum_{i=1}^{d} V_{i}(x) D u \circ \mathrm{d} z_{t}^{i} & & \text { on }[0, T] \times \mathbb{R}^{n}, \\
u(0, x) & =u_{0}(x) & & \text { on } \mathbb{R}^{n} .
\end{aligned}\right.
$$

First assume that $z$ is a smooth path and denote by $\phi$ the ODE flow

$$
\mathrm{d} \phi(t, x)=V(\phi(t, x)) \mathrm{d} z_{t}, \phi(0, x)=x \in \mathbb{R}^{n} .
$$

Then (at least formally) we see that the function $v(t, x):=u(t, \phi(t, x))$ solves the standard parabolic heat equation

$$
\left\{\begin{aligned}
\mathrm{d} v & =\sigma_{\phi}^{2}(t, x) D^{2} v \mathrm{~d} t & & \text { on }[0, T] \times \mathbb{R}^{n}, \\
v(0, x) & =u_{0}(x) & & \text { on } \mathbb{R}^{n},
\end{aligned} \text { where } \sigma_{\phi}^{2}(t, x):=\sigma^{2}(t, \phi(t, x)) .\right.
$$

An obvious idea for the case that $z$ is no longer a smooth path is to approximate $z$ by a sequence of smooth paths $\left(z^{n}\right)$. For each fixed $n \in \mathbb{N}$ one can solve the ODE flow $\phi^{n}$ (the ODE (21) with $z$ replaced by $z^{n}$ ) and subsequently the corresponding simple PDE (22) to arrive at the sequence of PDE solutions $\left(v^{n}\right)$. Since the flow $\phi^{n}$ will be a diffeomorphism we also know that

$$
u^{n}(t, x)=v^{n}\left(t,\left(\phi_{t}^{n}\right)^{-1}(x)\right)
$$

where $u^{n}$ denotes the solution of (20) where the driving signal $z$ is replaced by $z^{n}$. Obviously we expect that $\left(v^{n}\right)_{n}$ as well as $\left(\phi^{n}\right)_{n}$ converge as $n \rightarrow \infty$ : for $\left(v^{n}\right)_{n}$ this should follow from the robust approximations of operators from viscosity theory and for $\left(\phi^{n}\right)_{n}$ this should follow if we consider convergence in rough path metricrecall Section 2 and 3 on the problems caused by highly oscillatory driving signals $z$. If this holds, then (23) implies that $\left(u^{n}\right)_{n}$ converges to some $u$ and this function is a natural candidate for a solution. Of course, all the above was completely formal and the convergence can go wrong. However, with more care it can be made rigorous even for fully nonlinear operators; for the detailed argument see [12, 31].

Corollary 2 (Splitting for the Zakai SPDE). Denote by $\left\{P_{t}, t \geq 0\right\}$ the solution operator 
$\varphi \mapsto v$ where $v$ is the viscosity solution of $\mathrm{d} v=L\left(t, x, D v, D^{2} v\right) \mathrm{d} t, v(0, \cdot)=\varphi(\cdot)$

and by $\left\{Q_{s, t}, 0 \leq s \leq t\right\}$ the solution operator

$\varphi \mapsto v$ where $v$ is the SDE solution of $\mathrm{d} y=\Lambda(t, x, D v) \circ \mathrm{d} B_{t}, y(0, \cdot)=\varphi(\cdot)$.

Then for a.e. $\omega$

$$
u^{n}(t, x):=\prod_{i=0}^{\lfloor t / n\rfloor-1}\left[Q_{i / n, i / n+1 / n} \circ P_{1 / n}\right]\left(u_{0}(x)\right)
$$

converges locally uniformly (in $(t, x)$ ) as $n \rightarrow \infty$ to the unique solution $u$ of (19) given by Theorem 6 with $z_{t}=B_{t}(\omega) \equiv\left(1, B_{t}(\omega),\left(\int_{0}^{t} B \otimes \circ \mathrm{d} B\right)(\omega)\right)$.

\section{Pathwise optimal control}

Consider the SDE

$$
\mathrm{d} X_{t}=a\left(X_{t}, \alpha_{t}\right) \mathrm{d} t+b\left(X_{t}, \alpha_{t}\right) \circ \mathrm{d} B_{t}+c\left(X_{t}\right) \circ \mathrm{d} \tilde{B}_{t}
$$

where $t \mapsto \alpha_{t}$ is a path, $B$ and $\tilde{B}$ are multi-dimensional, independent Brownian motions and $(a, b, c)$ are (sufficiently regular) vector fields. In applications (engineering, economics, etc.) one often faces the problem that one can influence the evolution of $X$ by controlling the path $\alpha$. The aim is then to minimize a cost function (consisting of a terminal cost $g$ and a running cost $f$ ) of the form

$$
v(t, x)=\inf _{\alpha} \mathbb{E}\left[g\left(X_{T}^{t, x}\right)+\int_{t}^{T} f\left(X_{s}^{t, x}, \alpha_{s}\right) \mathrm{d} s \mid \tilde{B}\right] .
$$

It turns out that we can use the Bellman principle to describe the change in the cost function over time by a SPDE, the so-called Hamilton-Jacobi-Bellman (S)PDE. Indeed, a formal computation (see [21] for a rigorous derivation from basic principles) shows that after the time reversal $u(t, x):=v(T-t, x)$, we get a SPDE of the form

$$
\left\{\begin{aligned}
\left.\mathrm{d} u\right|_{t, x}+\inf _{\alpha}\left[\left.b\left(x, \alpha_{t}\right) D u\right|_{t, x}+\left.L_{\alpha} u\right|_{t, x}+f\left(x, \alpha_{t}\right)\right] \mathrm{d} t+\left.D u\right|_{t, x} \cdot c(x) \circ \mathrm{d} \tilde{B}_{t} & =0 & & \text { on }[0, T] \times \mathbb{R}^{n}, \\
u(0, x) & =g(x) & & \text { on } \mathbb{R}^{n},
\end{aligned}\right.
$$

where $L_{\alpha}$ is the linear differential operator with $(a, b)$. Using

Corollary 3. Let $z \in C^{0, p-v a r}\left([0, T], \mathbb{R}^{d}\right)$. The SPDE (25) is robust in rough path sense.

The proof is a slight modification of the proof of Theorem 6 since the usual comparison results from viscosity theory is stable under taking $\inf _{\alpha}$. 


\section{Weak splitting schemes for SPDEs}

In the previous sections we have concentrated on strong approximation of (partial) differential equations driven by random signals, i.e., on the approximation of the solution $y_{T}=y_{T}(\omega)$ of the rough or stochastic (partial) differential equation as a random variable, $\omega$-for- $\omega$ (resp. rough path by rough path). However, in many applications one is only interested in the law of the solution $y_{T}$ of the equation. Indeed, if the quantity of interest is just the expectation of a functional of the solution, say

$$
E\left[f\left(y_{T}\right)\right],
$$

then it is sufficient to only approximate the law of $y_{T}$. This corresponds to the notion of weak convergence of random variables, and hence schemes for approximating the law of the solution of a stochastic (partial) differential equations are referred to as weak schemes. More precisely, let us consider the solution $y_{T}$ of a stochastic differential equation defined on the Banach space $X$ (which is infinite-dimensional in the case of an SPDE) and let us consider a sequence of approximations $\bar{y}_{N}$ taking values in $X$ indexed by $N \in \mathbb{N}$. Fix a space of sufficiently regular test functions $f: X \rightarrow \mathbb{R}$ (classically chosen to be $C_{b}(X)$ in theoretical probability theory, but more flexibility is needed in numerics). Then we say that $\bar{y}_{N}$ converges to $y_{T}$ in the weak sense if for any test function $f$ we have

$$
E\left[f\left(\bar{y}_{N}\right)\right] \stackrel{N \rightarrow \infty}{\longrightarrow} E\left[f\left(y_{T}\right)\right] .
$$

In particular, note that weak schemes - unlike strong ones - do not have to operate on the same probability space as the true solution.

Of course, if the space of test functions is a subspace of the Lipschitz continuous functions, then strong convergence (i.e., convergence in $L^{1}(\Omega ; X)$ ) implies weak convergence, and the rate of weak convergence is at least as good as the rate of strong convergence. However, in many cases the weak rate of convergence is, in fact, much better than the strong one.

\section{Cubature on Wiener space}

For simplicity, let us concentrate on the finite-dimensional case first — we will come back to the infinite-dimensional (SPDE) setting at the end of this section. Consider the stochastic differential equation

$$
d y_{t}=V_{0}\left(y_{t}\right) d t+\sum_{i=1}^{d} V_{i}\left(y_{t}\right) \circ d B_{t}^{i}
$$


with $y_{0} \in \mathbb{R}^{e}$ fixed, $B$ denoting a $d$-dimensional standard Brownian motion and "o" indicating that the stochastic integral is understood in the Stratonovich sense. We furthermore introduce the notation $B_{t}^{0} \equiv t$ to simplify the presentation.

Assumption 3. We assume that the vector fields $V_{0}, \ldots, V_{d}: \mathbb{R}^{e} \rightarrow \mathbb{R}^{e}$ are $C^{\infty}$ bounded, i.e., they are smooth and all the derivatives are bounded (but not necessarily the functions themselves). Moreover, the test function $f$ is smooth and bounded.

Remark 1. Of course, these assumptions can be relaxed. For instance, the boundedness requirements can be removed by working with properly weighted norms $[22,45]$. Moreover, assuming a (hypo-)ellipticity condition for the vector fields, we can actually rely on the smoothing property of the diffusion equation and drop the smoothness assumption for the test function $f-$ at the cost of possibly having to work with non-uniform grids, see [53].

In order to derive appropriate weak splitting schemes for the equation (26), we first recall the short time behaviour of the solution using the stochastic Taylor expansion, see for instance [44]. By iterating the Ito-formula for the StratonovichSDE (26) $m$ times, we obtain

$f\left(y_{t}\right)=f\left(y_{0}\right)+\sum_{k=1}^{m} \sum_{\left(i_{1}, \ldots, i_{k}\right) \in\{0, \ldots, d\}^{k}} V_{i_{1}} \cdots V_{i_{k}} f\left(y_{0}\right) \int_{0<t_{1}<\cdots<t_{k}<t} \circ d B_{t_{1}}^{i_{1}} \cdots \circ d B_{t_{k}}^{i_{k}}+\mathscr{O}\left(t^{(m+1) / 2}\right)$,

where we iteratively use the geometrical notion $V f(x) \equiv \nabla f(x) \cdot V(x)$ for a function $f$ and a vector field $V$. We also denote

$$
B_{t}^{I}=B_{t}^{\left(i_{1}, \ldots, i_{k}\right)} \equiv \int_{0<t_{1}<\cdots<t_{k}<t} \circ d B_{t_{1}}^{i_{1}} \cdots \circ d B_{t_{k}}^{i_{k}}, \quad I=\left(i_{1}, \ldots, i_{k}\right) \in\{0, \ldots, d\}^{k} .
$$

Remark 2. We once again see that the short-time behaviour of the solution $y$ is controlled by the truncated signature.

Remark 3. As a matter of fact, sharper versions of (27) are possible, in so far that (27) ignores the different scaling of $t=B_{t}^{0}$ and $B_{t}^{1}, \ldots, B_{t}^{d}$. Once again, we refer to [53].

Remark 4. Of course, analogous stochastic Taylor expansions can also be formulated in terms of the Ito integral, which would then lead to the Ito-signature. We prefer the geometrically more intuitive Stratonovich versions in this chapter.

This motivates the following methodology for constructing higher order weak approximation schemes termed the $O D E$ method (originally introduced as cubature on Wiener space by [53] and, independently, [46]).

Theorem 7. In the setting of Assumption 3, we are given a time-grid $0=t_{0}<t_{1}<$ $\cdots<t_{N}=T$ with corresponding increments $\Delta t_{i}, i=1, \ldots, N$. Let $W_{i}:\left[0, \Delta t_{i}\right] \rightarrow$ $\mathbb{R}^{d+1}$ be a $(d+1)$-dimensional path of bounded variation satisfying 


$$
\forall 0 \leq k \leq m, I \in\{0, \ldots, d\}^{k}: E\left[B_{\Delta t_{i}}^{I}\right]=E\left[W_{i}^{I}\left(\Delta t_{i}\right)\right] .
$$

Moreover, let $W:[0, T] \rightarrow \mathbb{R}^{d+1}$ be the bounded-variation process obtained by concatenating the processes $W_{1}, \ldots, W_{N}$. Finally, let $\bar{y}_{N} \equiv y_{T}(W)$ be defined as the solution of the ODE

$$
\frac{d y(W)_{t}}{d t}=V_{0}\left(y(W)_{t}\right) \dot{W}_{t}^{0}+\sum_{i=1}^{d} V_{i}\left(y(W)_{t}\right) \dot{W}_{t}^{i}
$$

formally obtained from (26) by replacing $B$ by $W$. Then there is a constant $C>0$ such that

$$
\mid E\left[f\left(y_{T}\right)\right]-E\left[f\left(y(W)_{T}\right] \mid \leq C\left(\max _{i=1, \ldots, N} \Delta t_{i}\right)^{(m-1) / 2} .\right.
$$

Proof. We do not give a detailed proof, as the underlying argument is quite standard in numerical analysis. Indeed, by (27) the local error of the approximation is of order $\left(\Delta t_{i}\right)^{(m+1) / 2}$. Thus, by summing up the local errors we obtain that the global error is of order $\left(\max _{i} \Delta t_{i}\right)^{(m-1) / 2}$

Remark 5. The above theorem is somewhat imprecise, as the constant $C$ depends on $T, f$, the vector fields $V_{0}, \ldots, V_{d}$ and the method of constructing the processes $W_{1}, \ldots, W_{N}$, but not on the grid. E.g., in the case of the Ninomiya-Victoir method introduced below, $C$ will only depend on $T, f, V_{0}, \ldots, V_{d}$.

\subsection{The Ninomiya-Victoir splitting}

If liberally interpreted - e.g., for Euler schemes, when the path $W$ is actually a stepfunction - Theorem 7 encompasses a large class of discretization schemes for the stochastic differential equation (26). In particular, it allows for a simple construction of stochastic splitting schemes, as we shall exemplify by the arguably most popular version, the Ninomiya-Victoir scheme [58]. In that case, the paths of the process $W$ are axis-paths, i.e., the paths are continuous and piecewise-parallel to the axis in $\mathbb{R}^{d+1}$, similar to the construction used in Definition 3, see (9). More precisely, choose $1 \leq i \leq N$ and a sequence of independent (of all other sources of randomness) random variables $\Lambda_{i}, i=1, \ldots, N$ with $P\left(\Lambda_{i}=1\right)=P\left(\Lambda_{i}=-1\right)=1 / 2$. Construct a process $W_{i}$ on $\left[0, \Delta t_{i}\right]$ in the following way: set $\delta_{i} \equiv \Delta t_{i} /(d+1)$ and when $\Lambda_{i}=+1$, set

$$
\dot{W}_{i}(t)= \begin{cases}\Delta t_{i} / \delta_{i} e_{0}, & 0 \leq t<1 / 2 \delta_{i}, \\ \Delta B_{i}^{j} / \delta_{i} e_{j}, & (1 / 2+(j-1)) \delta_{i} \leq t<(1 / 2+j) \delta_{i}, 1 \leq j \leq d, \\ \Delta t_{i} / \delta_{i} e_{0}, & \Delta t_{i}-1 / 2 \delta_{i} \leq t \leq \Delta t_{i},\end{cases}
$$

where we recall that $\Delta B_{i}^{j} \equiv B_{t_{i}}^{j}-B_{t_{i-1}}^{j}$ and where we denote by $\left(e_{0}, e_{1}, \ldots, e_{d}\right)$ the standard basis of $\mathbb{R}^{d+1}$. In the other case $\left(\Lambda_{i}=-1\right)$, we define $W_{i}$ by 


$$
\dot{W}_{i}(t)= \begin{cases}\Delta t_{i} / \delta_{i} e_{0}, & 0 \leq t<1 / 2 \delta_{i}, \\ \Delta B_{i}^{d-j+1} / \delta_{i} e_{d-j+1}, & (1 / 2+(j-1)) \delta_{i} \leq t<(1 / 2+j) \delta_{i}, 1 \leq j \leq d, \\ \Delta t_{i} / \delta_{i} e_{0}, & \Delta t_{i}-1 / 2 \delta_{i} \leq t \leq \Delta t_{i} .\end{cases}
$$

As in the general construction, the independent processes $W_{1}, \ldots, W_{N}$ are then concatenated to form the process $W$ defined on $[0, T]$.

Inserting the process $W$ just constructed into the general methodology (29), we see that the Ninomiya-Victoir method boils down to solving the ODEs driven by the individual vector fields $V_{0}, \ldots, V_{d}$ on $\mathbb{R}^{e}$. Indeed, let $e^{s V_{i}} x$ denote the flow associated to the vector field $V_{i}$ at time $s$, i.e., $e^{s V_{i}} x=z(s)$ solution to

$$
\dot{z}(t)=V_{i}(z(t)), \quad z(0)=x \in \mathbb{R}^{e},
$$

then the solution $\bar{y}_{l} \equiv y(W)_{t_{l}}, l=0, \ldots, N$, of (29) for the Ninomiya-Victoir process $W$ satisfies $\bar{y}_{0}=y_{0}$ and

$$
\bar{y}_{l}= \begin{cases}e^{\frac{\Delta t_{l}}{2} V_{0}} e^{\Delta B_{l}^{d} V_{d}} \cdots e^{\Delta B_{l}^{1} V_{1}} e^{\frac{\Delta t_{l}}{2} V_{0}} \bar{y}_{l-1}, & \Lambda_{l}=+1 \\ e^{\frac{\Delta t_{l}}{2} V_{0}} e^{\Delta B_{l}^{1} V_{1}} \cdots e^{\Delta B_{l}^{d} V_{d}} e^{\frac{\Delta t_{l}}{2} V_{0} \bar{y}_{l-1}}, & \Lambda_{l}=-1\end{cases}
$$

$l=1, \ldots, N$. This explains from the SDE side, why we consider the NinomiyaVictoir scheme a stochastic splitting scheme for the SDE (26).

Theorem 8. Under Assumption 3, the Ninomiya-Victoir scheme is a weak scheme of second order, i.e., there is a constant $C>0$ (depending on $T, f, V_{0}, \ldots, V_{d}$, but not on the grid) such that

$$
\mid E\left[f\left(y_{T}\right)\right]-E\left[f\left(y(W)_{T}\right] \mid \leq C\left(\max _{i=1, \ldots, N} \Delta t_{i}\right)^{2} .\right.
$$

Proof. We show that $E\left[S_{0, t}^{5}(B)\right]=E\left[S_{0, t}^{5}(W)\right]$ for $t=\Delta t_{i}$ and any $W=W_{i}$, which implies the conclusion by Theorem 7 .

Let us first consider the (Stratonovich) signature of the Brownian motion. By the construction of the Stratonovich integral in terms of the Ito integral, we have

$$
\begin{aligned}
B_{t}^{\left(i_{1}, \ldots, i_{k}\right)}=\int_{0<t_{1}<\cdots<t_{k}<t} \circ d B_{t_{1}}^{i_{1}} \cdots \circ d B_{t_{k}}^{i_{k}} \\
= \begin{cases}\int_{0}^{t} B_{t_{k}}^{\left(i_{1}, \ldots, i_{k-1}\right)} d B_{t_{k}}^{i_{k}}+\frac{1}{2} \int_{0}^{t} B_{s}^{\left(i_{1}, \ldots, i_{k-2}\right)} d s \delta_{i_{k} i_{k-1}}, & i_{k} \neq 0, \\
\int_{0}^{t} B_{t_{k}}^{\left(i_{1}, \ldots, i_{k-1}\right)} d B_{t_{k}}^{i_{k}}, & i_{k}=0 .\end{cases}
\end{aligned}
$$

Using the Ito isometry, the expectation of the iterated Stratonovich integral is iteratively given by

$$
E\left[B_{t}^{\left(i_{1}, \ldots, i_{k}\right)}\right]= \begin{cases}\frac{1}{2} \int_{0}^{t} E\left[B_{s}^{\left(i_{1}, \ldots, i_{k-2}\right)}\right] d s \delta_{i_{k} i_{k-1},}, & i_{k} \neq 0 \\ \int_{0}^{t} E\left[B_{s}^{\left(i_{1}, \ldots, i_{k-1}\right)}\right] d s, & i_{k}=0 .\end{cases}
$$


As regards the Ninomiya-Victoir cubature formula defined above, we see that

$$
\dot{W}_{i}^{j}(t)=\Delta B_{i}^{j} / \delta_{i} \mathbf{1}_{A_{i}^{j}}(t), \quad j=0, \ldots, d, \quad i=1, \ldots, N,
$$

where we tacitly let $\Delta B_{i}^{0}=\Delta t_{i}$ and define the set $A_{i}^{j}$ by

$$
\begin{gathered}
A_{i}^{0}=\left[0,1 / 2 \delta_{i}\left[\cup\left[\Delta t_{i}-1 / 2 \delta_{i}, \Delta t_{i}\right],\right.\right. \\
A_{i}^{j}=\left\{\begin{array}{ll}
{\left[(j-1 / 2) \delta_{i},(j+1 / 2) \delta_{i}\right],} & \Lambda_{i}=+1, \\
{\left[(d-j+1 / 2) \delta_{i},(d-j+3 / 2) \delta_{i}\right],} & \Lambda_{i}=-1,
\end{array} \quad j=1, \ldots, d .\right.
\end{gathered}
$$

So we have the general formula

$E\left[W_{i}^{\left(i_{1}, \ldots, i_{k}\right)}\left(\Delta t_{i}\right)\right]=E\left[\Delta B_{i}^{i_{1}} \cdots \Delta B_{i}^{i_{k}}\right] E\left[\int_{0<t_{1}<\cdots<t_{k}} \mathbf{1}_{A_{i}^{i_{1}}}\left(t_{1}\right) \cdots \mathbf{1}_{A_{i}^{i_{k}}}\left(t_{k}\right) d t_{1} \cdots d t_{k}\right]$,

where the last expectation is necessary due to the random choice of intervals above, and, in fact, only involves the two alternatives $\Lambda_{i}= \pm 1$.

The verification of the theorem now boils down to a simple, but tedious exercise. For instance, for multi-indices of length 3 , we see that the only non-zero components of the expectation of the signature restricted to multi-indices of length 3 for either $B$ and $W_{i}$ are

$$
\begin{aligned}
& E\left[B_{\Delta t_{i}}^{(0,0,0)}\right]=\frac{\Delta t_{i}^{3}}{6}=E\left[W_{i}^{(0,0,0)}\left(\Delta t_{i}\right)\right], \\
& E\left[B_{\Delta t_{i}}^{(0, j, j)}\right]=\frac{\Delta t_{i}^{2}}{4}=E\left[W_{i}^{(0, j, j)}\left(\Delta t_{i}\right)\right], \\
& E\left[B_{\Delta t_{i}}^{(j, j, 0)}\right]=\frac{\Delta t_{i}^{2}}{4}=E\left[W_{i}^{(j, j, 0)}\left(\Delta t_{i}\right)\right],
\end{aligned}
$$

$j=1, \ldots, d$,

\section{A path-wise interpretation of the Ninomiya-Victoir splitting scheme}

Interpreting the Ninomiya-Victoir scheme in the Lie/Strang splitting picture drawn in (9) and below, we define functions $a^{\Delta t_{i}}, b_{1}^{\Delta t_{i}}, \ldots, b_{d}^{\Delta t_{i}}$ on the interval $\left[0, \Delta t_{i}\right]$ by 


$$
\begin{gathered}
\dot{a}^{\Delta t_{i}}(t)=\frac{\Delta t_{i}}{\delta_{i}} \mathbf{1}_{\left[0, \delta_{i} / 2[\right.}(t), \\
\dot{b}_{j}^{\Delta t_{i}}(t)=\frac{\Delta B_{i}^{j}}{\delta_{i}} \mathbf{1}_{\left[(1 / 2+j-1) \delta_{i},(1 / 2+j) \delta_{i}[\right.}(t), \quad j=1, \ldots, d, \\
\dot{c}^{\Delta t_{i}}(t)=\frac{\Delta t_{i}}{\delta_{i}} \mathbf{1}_{\left[\Delta t_{i}-\delta_{i} / 2, \Delta t_{i}\right]}(t) .
\end{gathered}
$$

After concatenating these paths, we could immediately construct a Lie-type splitting following Definition 3 (in fact, we would not need to split the time component in the $a$ and $c$ paths) or a Strang-type splitting. However, taking the scaling of Brownian motion into account, we realize that we need to take care of Lie brackets of order up to 5 in order to obtain a high order scheme. Hence, we need even more "reorderings" than in the ordinary Strang splitting. Thus, we further define paths

$$
\widetilde{b}_{j}^{\dot{\Delta} t_{i}}(t)=\frac{\Delta B_{i}^{j}}{\delta_{i}} \mathbf{1}_{\left[(1 / 2+d-j) \delta_{i},(1 / 2+d-j+1) \delta_{i}[(t), \quad j=1, \ldots, d .\right.} .
$$

The two alternatives (30a) and (30b) of $W_{i}(t)$ are then given by

$$
W_{i}(t)=\left(a^{\Delta t_{i}}(t)+c^{\Delta t_{i}}(t)\right) e_{0}+\sum_{j=1}^{d}\left(b_{j}^{\Delta t_{i}}(t) \mathbf{1}_{\Lambda_{i}=+1}+\widetilde{b}_{j}^{\Delta t_{i}}(t) \mathbf{1}_{\Lambda_{i}=-1}\right) e_{j},
$$

and the corresponding splitting scheme is indeed given by (31), taking into account that the solutions of the ODEs driven by $b_{j}^{\Delta t_{i}}$ and $\widetilde{b}_{j}^{\Delta t_{i}}$ eventually coincide at time $\Delta t_{i}$.

\section{The Ninomiya-Victoir scheme as a splitting scheme for PDEs}

It is well-known that the function $u(t, y) \equiv E\left[f\left(y_{t}\right)\right]$ with $y_{0}=y$ satisfies the linear Cauchy problem

$$
\frac{\partial}{\partial t} u(t, y)=L u(t, y), \quad u(0, y)=f(y),
$$

for $t>0$ and $y \in \mathbb{R}^{e}$, respectively. Here, the partial differential operator $L$ is defined by

$$
L g(y)=V_{0} g(y)+\frac{1}{2} \sum_{i=1}^{d} V_{i}^{2} g(y),
$$

where we recall that for any vector field $V: \mathbb{R}^{e} \rightarrow \mathbb{R}^{e}$ and any smooth function $g: \mathbb{R}^{e} \rightarrow \mathbb{R}$ we set $V g(y) \equiv \nabla g(y) \cdot V(y)$. Iterating this procedure also defines $V^{2} g$, with $V^{2}$ a second order differential operator. Hence, for any weak approximation $\bar{y}_{N}$ of $y_{T}$, we have that

$$
E\left[f\left(\bar{y}_{N}\right)\right] \approx E\left[f\left(y_{T}\right)\right]=u\left(T, y_{0}\right),
$$


and the order of the weak approximation is the order of the approximation in the solution of the PDE (32). In semi-group notation, we can denote the solution operator associated to $L$ by $P_{t} \equiv \exp (t L)$, i.e.,

$$
u(t, y)=P_{t} f(y) .
$$

Remark 6. Obviously, solving the SDE (26) is only one step for the solution of the PDE (32): in addition, one needs to approximate the expectation in (34). In principle, for the Ninomiya-Victoir method this is a numerical integral in dimension $d \times N$. As this dimension is typically quite high, one usually resorts to Monte Carlo or Quasi Monte Carlo methods for computing the integral. Numerically, the computational cost of the integration step is often much higher than the computational cost of the discretization of the SDE, as the rate of convergence of the integration schemes is only $\frac{1}{2}$ (for Monte Carlo) or (at best) 1 (for Quasi Monte Carlo). Nonetheless, higher order weak approximation methods can reduce the overall computational cost considerably as compared to low order methods, partly because they actually lead to a considerable reduction of the dimension of the integration problem in the second step. The advantages of using higher order schemes have been observed in many numerical studies, for instance [58, 2, 4, 45].

Remark 7. As compared to classical numerical solvers for the Cauchy problem (32), the stochastic approximation scheme presented here has some very different features. On the one hand, most standard numerical methods such as finite element or finite difference schemes produce approximate solutions $u(t, y)$ for all values of $t$ and $y$ simultaneously - within a certain region in time and space, and up to interpolation. On the other hand, using the stochastic representation (34), one only obtains an approximation of $u(t, y)$ for one particular $t$ and one particular $y$. Moreover, the stochastic method crucially relies on the performance of the (Q)MC approximation for the expected values, and shares its strengths and weaknesses. Hence, for lowdimensional problems classical numerical PDE solvers are typically more efficient, whereas for high dimensions $e \gg 1$, the stochastic method is competitive or superior, as it does not suffer from the curse of dimensionality.

In light of (32), the question arises whether the Ninomiya-Victoir scheme can be naturally associated with a (PDE) splitting scheme for (32). To this end let us first consider the situation when there is only one time-step. Let $Q_{t}^{i}, i=1, \ldots, d$, be the semi-groups corresponding to the second order differential operators $\frac{1}{2} V_{i}^{2}$, $i=1, \ldots, d$, respectively. Formally, we write $Q_{t}^{i}=e^{\frac{t}{2} V_{i}^{2}}$. Moreover, we denote by $Q_{t}^{0}$ the semi-group associated with the operator $V_{0}$, i.e.,

$$
Q_{t}^{0} f(x)=f\left(e^{t V_{0}} x\right)
$$

While the correspondence between $e^{t V_{0}}$ and $Q_{t}^{0}$ is obvious, we note that $Q_{t}^{i}$ is in some sense the expectation of $e^{B_{t}^{i} V_{i}}$. More precisely, stochastic Taylor expansion shows that for any $C^{\infty}$-bounded test function $f$ and any initial value $y \in \mathbb{R}^{e}$ we have 
The splitting method for SPDEs

$$
Q_{t}^{i} f(y)=E\left[f\left(e^{B_{t}^{i} V_{i}} y\right)\right]
$$

Hence, in the case with only one time-step (with $\Delta t=T, \Delta B=B_{T}$ ) we obtain that

$$
\begin{aligned}
E\left[f\left(\bar{y}_{1}\right)\right] & =\frac{1}{2} E\left[f\left(e^{\frac{\Delta t}{2} V_{0}} e^{\Delta B^{d} V_{d}} \cdots e^{\Delta B^{1} V_{1}} e^{\frac{\Delta t}{2} V_{0}} y\right)\right]+\frac{1}{2} E\left[f\left(e^{\frac{\Delta t}{2} V_{0}} e^{\Delta B^{1} V_{1}} \cdots e^{\Delta B^{d} V_{d}} e^{\frac{\Delta t}{2} V_{0}} y\right)\right] \\
& =\frac{1}{2} Q_{\Delta t / 2}^{0} Q_{\Delta t}^{d} \cdots Q_{\Delta t}^{1} Q_{\Delta t / 2}^{0} f(y)+\frac{1}{2} Q_{\Delta t / 2}^{0} Q_{\Delta t}^{1} \cdots Q_{\Delta t}^{d} Q_{\Delta t / 2}^{0} f(y) \equiv Q_{\Delta t} f(y) .
\end{aligned}
$$

Note that the weight " $\frac{1}{2}$ " comes from the probability $\frac{1}{2}$ to choose either of the two alternatives in (31).

Iterating this construction along a discretization of the time interval $[0, T]$ as above, we recover a well-known splitting scheme from the PDE literature, sometimes referred to as "symmetrically weighted sequential splitting" scheme, see [18]. In terms of the solution operator $P_{t}=\exp (t L)$, Theorem 8 thus says that

$$
\left|P_{T} f(y)-Q_{\Delta t_{N}} \cdots Q_{\Delta t_{1}} f(y)\right| \leq C\left(\max _{i=1, \ldots, N} \Delta t_{i}\right)^{2} .
$$

\section{The Ninomiya-Victoir stochastic splitting for SPDEs}

The stochastic splitting methodology introduced above can be directly generalized to the infinite-dimensional case, i.e., to the case of SPDEs instead of SDEs. This was first done by Bayer and Teichmann [5] for the abstract formulation of Theorem 7 under strong regularity conditions. Later on, Dörsek and Teichmann [22] have given a careful analysis of the Ninomiya-Victoir splitting and other splitting techniques for weak approximation of SPDEs under weaker assumptions. We will mainly follow their approach here.

Consider a stochastic partial differential equation of the form

$$
d y_{t}=\left(A y_{t}+V\left(y_{t}\right)\right) d t+\sum_{i=1}^{d} V_{i}\left(y_{t}\right) d B_{t}^{i}, \quad y_{0} \in X,
$$

that is we assume that the stochastic fluctuation only depend on $y_{t}$, but not on derivatives of $y_{t}$. The state space $X$ of the equation (36) is assumed to be a separable Hilbert space and the vector fields $V, V_{1}, \ldots, V_{d}: X \rightarrow X$ are Frechet-differentiable and Lipschitz continuous, whereas the operator $A: \mathscr{D}(A) \subset X \rightarrow X$ is the generator of a strongly continuous, pseudo-contractive semigroup on $X$ - more regularity conditions on the coefficients are deferred until later. Then, a mild solution $y_{t}$ of the SPDE exists. For details of the solution theory of this class of SPDEs we refer to the monograph [19].

As mild solutions to SPDEs of the form (36) are generally not semi-martingales, we cannot re-write (36) in Stratonovich form from the beginning, but have to work 
with the Ito formulation. Nonetheless, if we use the Ninomiya-Victoir stochastic splitting approach, all the resulting (simpler) SPDEs can, in fact, be written in Stratonovich form, hence we proceed just as above. Indeed, define

$$
e^{s V_{i}} y=z_{s}, \text { where } \dot{z}_{t}=V_{i}\left(z_{t}\right), z_{0}=y \in X, \quad i=1, \ldots, d .
$$

Moreover, set $V_{0}(y) \equiv V(y)-\frac{1}{2} \sum_{i=1}^{d} D V_{i}(y) \cdot V_{i}(y), y \in X$, which would precisely correspond to the Stratonovich drift if it actually were to exist, and define $e^{s\left(A+V_{0}\right)} y$ analogously, i.e., as solution $z_{s}$ at time $s$ of the Cauchy problem

$$
\frac{\partial}{\partial t} z_{t}=A z_{t}+V_{0}\left(z_{t}\right), \quad z_{0}=y \in X
$$

which may be represented in terms of the semi-group $\exp (t A)$ generated by $A$ as

$$
e^{s\left(A+V_{0}\right)} y=z_{s}=\exp (s A) y+\int_{0}^{s} \exp ((s-t) A) V_{0}\left(z_{t}\right) d t .
$$

Now we can define the Ninomiya-Victoir splitting essentially as in (31), i.e., we set $\bar{y}_{0}=y_{0}$ and

$$
\bar{y}_{l}= \begin{cases}e^{\frac{\Delta t_{l}}{2}\left(A+V_{0}\right)} e^{\Delta B_{l}^{d} V_{d}} \cdots e^{\Delta B_{l}^{1} V_{1}} e^{\frac{\Delta t_{l}}{2}\left(A+V_{0}\right)} \bar{y}_{l-1}, & \text { with prob. } \frac{1}{2}, \\ e^{\frac{\Delta t_{l}}{2}\left(A+V_{0}\right)} e^{\Delta B_{l}^{1} V_{1}} \cdots e^{\Delta B_{l}^{d} V_{d}} e^{\frac{\Delta t_{l}}{2}\left(A+V_{0}\right)} \bar{y}_{l-1}, & \text { else }\end{cases}
$$

$l=1, \ldots, N$.

We formulate assumptions given in [22], which can, in fact, be weakened using suitably weighted spaces.

Assumption 4. Consider the coefficients $A, V, V_{1}, \ldots, V_{d}$ of the SPDE (36) and a function $f: X \rightarrow \mathbb{R}$. We assume that

- $A: \mathscr{D}(A) \subset X \rightarrow X$ generates a strongly continuous, pseudo-contractive semigroup on $X$ and has a compact resolvent.

- $V, V_{1}, \ldots, V_{d} \in C^{6}(X, X)$ and have bounded derivatives.

- $V, V_{1}, \ldots, V_{d}$ are Lipschitz when considered as maps $\mathscr{D}\left(A^{l}\right) \rightarrow \mathscr{D}\left(A^{l}\right), l=1, \ldots, 5$, where $\mathscr{D}\left(A^{l}\right)$ is equipped with the graph norm, i.e., the Hilbert norm given by $\|x\|_{\mathscr{D}\left(A^{l}\right)}^{2} \equiv\|x\|_{X}^{2}+\sum_{k=1}^{l}\left\|A^{k} x\right\|_{X}^{2}$.

- $f \in C_{b}^{6}(X)$.

Theorem 9 ([22, Cor. 7.11, Th. 7.20]). Under Assumption 4, there is a constant $C$ such that

$$
\left|E\left[f\left(y_{T}\right)\right]-E\left[f\left(\bar{y}_{N}\right)\right]\right| \leq C\left(\max _{i=1, \ldots, N} \Delta t_{i}\right)^{2} .
$$

Remark 8 . The theorem can also be re-formulated completely deterministically in the fashion of (35), i.e., as a deterministic splitting method for a PDE on an infinitedimensional state space. This is the version actually given in [22]. 


\section{Applications of weak schemes in financial engineering}

Given a financial model of the form (26), where $y_{t}$ could be a (one- or multidimensional) vector of asset (forward) prices, or a vector of asset prices and stochastic volatilities, or the individual factors of a multi-factor model, .... Disregarding financial technicalities (discounting, change to the pricing measure), we are concerned in computing a European option price with payoff function $f: \mathbb{R}^{e} \rightarrow \mathbb{R}$ and maturity $T$, i.e., our quantity of interest is

$$
E\left[f\left(y_{T}\right)\right]
$$

This simple option pricing problem is mostly relevant for calibration purposes, i.e., for identifying the model parameters which provide the best fit to the observed market prices. Hence, the speed of the pricing algorithm is extremely important for this application - more important than accuracy, due to the usually non negligible model error. ${ }^{5}$ In this section we give an overview of some successful applications of weak stochastic splitting methods in this context. We begin with two numerical studies on the performance of the Ninomiya-Victoir scheme for two popular (finitedimensional) models often used in financial engineering - the SABR model and the CEV model, a special case of the former $[2,4]$. Then we report the performance of the Ninomiya-Victoir method in an actual calibration routine for yet another related model, the double-mean-reverting model, [3]. Finally, we present the performance of the weak stochastic splitting method for SPDEs, again in the context of a calibration problem, this time for an interest rate model, the Heath-Jarrow-Morton model, see [23].

\section{Option pricing in high dimensions}

The SABR model is a prominent example of a stochastic interest rate model. We consider the following generalizations of the classical SABR model (cf. [2]).

$$
\begin{aligned}
& d y_{1}(t)=a y_{2}(t)^{\alpha} y_{1}(t)^{\beta} d B_{t}^{1}, \\
& d y_{2}(t)=\kappa\left(\theta-y_{2}(t)\right) d t+b y_{2}(t)\left(\rho d B_{t}^{1}+\sqrt{1-\rho^{2}} d B_{t}^{2}\right),
\end{aligned}
$$

with $X_{1}(0)=x_{1}$ and $X_{2}(0)=x_{2}$. We assume that the parameters satisfy $\frac{1}{2} \leq \beta \leq 1$, $\theta, \kappa \geq 0, \alpha>0, a, b>0,-1<\rho<1$. Here, the first component $y_{1}(t)$ models the (discounted) price of a stock, and $y_{2}(t)$ can be interpreted as some kind of stochastic volatility. In fact, the dynamics of $y_{1}$ depend in a nonlinear way on $y_{1}$ (local volatility) and on a second stochastic process $y_{2}$ (stochastic volatility). Hence, models

${ }^{5}$ That is, even without any numerical error, it is generally not possible to obtain a perfect fit to market prices, due to the model limitations. 
of this kind are known as stochastic local volatility models. Moreover, note that this model can be easily generalized to the multi-asset case by just adding new processes $y$ with the same kind of dynamics, but driven by correlated Brownian motions for every new asset to be included in the model, see [2] for more details. We concentrate on the one-asset case for ease of presentation.

For the SABR model (38), the Stratonovich drift vector field and the diffusion vector fields are given by

$V_{0}(y)=\left(\begin{array}{c}-\frac{1}{2} a^{2} \beta y_{2}^{2 \alpha} y_{1}^{2 \beta-1}-\frac{1}{2} \alpha a b \rho y_{2}^{\alpha} y_{1}^{\beta} \\ \kappa \theta-\left(\kappa+\frac{1}{2} b^{2}\right) y_{2}\end{array}\right), \quad V_{1}(y)=\left(\begin{array}{c}a y_{2}^{\alpha} y_{1}^{\beta} \\ b \rho y_{2}\end{array}\right), \quad V_{2}(y)=\left(\begin{array}{c}0 \\ b \sqrt{1-\rho^{2}} y_{2}\end{array}\right)$.

Quite typically for models in financial engineering, the Stratonovich drift vector field is considerably more complicated than the Ito drift vector field or the diffusion vector fields which can be seen as a consequence of the Stratonovich correction $V_{0}=V-\frac{1}{2} \sum_{i=1}^{d} D V_{i} \cdot V_{i}$, noting that models in financial engineering are typically formulated in Ito form. As a consequence, it is not surprising that we have explicit formulas for the flow of the diffusion vector field, but not for the flow of the Stratonovich vector field $V_{0}$. In fact, we have

$$
\mathrm{e}^{s V_{1}} y=\left(\begin{array}{c}
g_{1}(s) \\
y_{2} \exp (b \rho s)
\end{array}\right), \quad \mathrm{e}^{s V_{2}} y=\left(\begin{array}{c}
y_{1} \\
y_{2} \exp \left(b \sqrt{1-\rho^{2}} s\right)
\end{array}\right)
$$

where

$$
g_{1}(s)= \begin{cases}{\left[(1-\beta) a y_{2}^{\alpha} \frac{\mathrm{e}^{\alpha b \rho s}-1}{\alpha b \rho}+y_{1}^{1-\beta}\right]_{+}^{1 /(1-\beta)},} & \frac{1}{2} \leq \beta<1, \\ y_{1} \exp \left(a x_{2}^{\alpha} \frac{\mathrm{e}^{\alpha b \rho s}-1}{\alpha b \rho}\right), & \beta=1 .\end{cases}
$$

Of course, it is possible to compute $e^{s V_{0}} y$ numerically, but for efficiency (and often also for geometrical reasons) it is preferable to have explicit solutions whenever possible. ${ }^{6}$ We therefore propose to slightly adjust the Ninomiya-Victoir splitting formula, taking the Stratonovich drift correction into account. That means, we replace the splitting $L=V_{0}+\frac{1}{2} \sum_{i=1}^{d} V_{i}^{2}$ by the splitting

$$
L=V_{0}^{(\gamma)}+\frac{1}{2} \sum_{i=1}^{d}\left(V_{i}^{2}+2 \gamma_{i} V_{i}\right), \text { where } V_{0}^{(\gamma)}=V_{0}-\sum_{i=1}^{d} \gamma_{i} V_{i}
$$

and $\gamma \in \mathbb{R}^{d}$ is chosen such that the flow of $V_{0}^{(\gamma)}$ has an explicit solution. Note that $\frac{1}{2} V_{i}^{2}+\gamma_{i} V_{i}$ corresponds to the stochastic equation

$$
d z_{t}=\gamma_{i} V_{i}\left(z_{t}\right) d t+V_{i}\left(z_{t}\right) \circ d B_{t}^{i}=V_{i}\left(z_{t}\right) \circ d\left(B_{t}^{i}+\gamma_{i} t\right),
$$

\footnotetext{
${ }^{6}$ By which we do not mean difficult-to-evaluate series expansions, Bessel functions or similar solutions. Instead, we mean formulas with comparable complexity to the vector fields themselves.
} 
i.e., the stochastic weak splitting scheme actually looks exactly like the standard Ninomiya-Victoir scheme (31), but with $\Delta B_{l}^{i}$ replaced by $\Delta B_{l}^{i}+\gamma_{i} \Delta t_{l}$ and $V_{0}$ replaced by $V_{0}^{(\gamma)}$ :

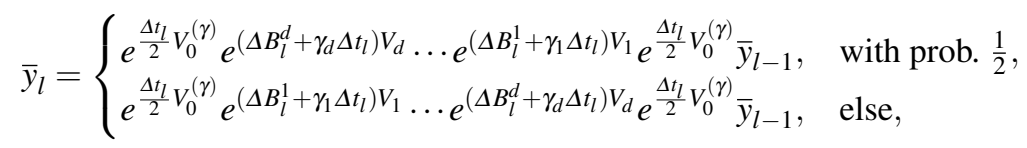

see [2]. As a matter of fact, we can easily find such a choice of $\gamma$ for the generalized SABR model given by

$$
\gamma_{1}=-\frac{1}{2} \alpha b \rho, \quad \gamma_{2}=\frac{\alpha b \rho^{2}-2 \kappa / b-b}{2 \sqrt{1-\rho^{2}}},
$$

leading to

$$
V_{0}^{(\gamma)}(y)=\left(\begin{array}{c}
-\frac{1}{2} a^{2} \beta y_{2}^{2 \alpha} y_{1}^{2 \beta-1} \\
\kappa \theta
\end{array}\right), \quad e^{s V_{0}^{(\gamma)}} y=\left(\begin{array}{c}
g_{0}(s ; y) \\
\kappa \theta s+y_{2}
\end{array}\right)
$$

with

$$
g_{0}(s ; y)= \begin{cases}\left(-a^{2} \beta(1-\beta) P(s ; y)+y_{1}^{2(1-\beta)}\right)_{+}^{\frac{1}{2(1-\beta)}}, & \frac{1}{2}<\beta<1, \\ y_{1} \exp \left(-\frac{1}{2} a^{2} P(s ; y)\right), & \beta=1, \\ -\frac{1}{4} a^{2} P(s ; y)+y_{1}, & \beta=\frac{1}{2},\end{cases}
$$

and

$$
P(s ; y)=\frac{1}{(2 \alpha+1) \kappa \theta}\left(\left(\kappa \theta s+y_{2}\right)^{2 \alpha+1}-y_{2}^{2 \alpha+1}\right) .
$$

Finally, let us present the results from one of the numerical experiments in [2] with real-world data. The parameters there were chosen to be $\beta=1.0, \theta=0.3$, $\kappa=2.0, \alpha=0.5, a=1.0, b=0.5, \rho=-0.7, y_{1}=1.0$ and $y_{2}=0.2$. The option has strike price $K=1.05$ and time to maturity $T=1.0$ years. The estimated "true result" is 0.1767505855 . Note that the expectation is computed by quasi Monte Carlo based on Sobol numbers.

In Figure 4 the discretization error is plotted against the number of time steps for three different schemes: the standard Euler scheme, the classical Ninomiya-Victoir splitting (31) (where $e^{s V_{0}}$ is computed by a standard second order Taylor expansion) and our adjusted Ninomiya-Victoir scheme (40). We clearly see the second order weak convergence of the Ninomiya-Victoir scheme in both variants as compared to the first order weak convergence of the standard Euler scheme.

Note that the error of the Euler scheme changes its sign at around two time steps, which explains the visible kink of the error of the Euler scheme in Figure 4. We consider this a numerical artifact which we disregard for the comparison.

In Table 1 the computational times are reported for the generalized SABR model. Here, the computational parameter $N$ (the number of uniform time-steps) is chosen 


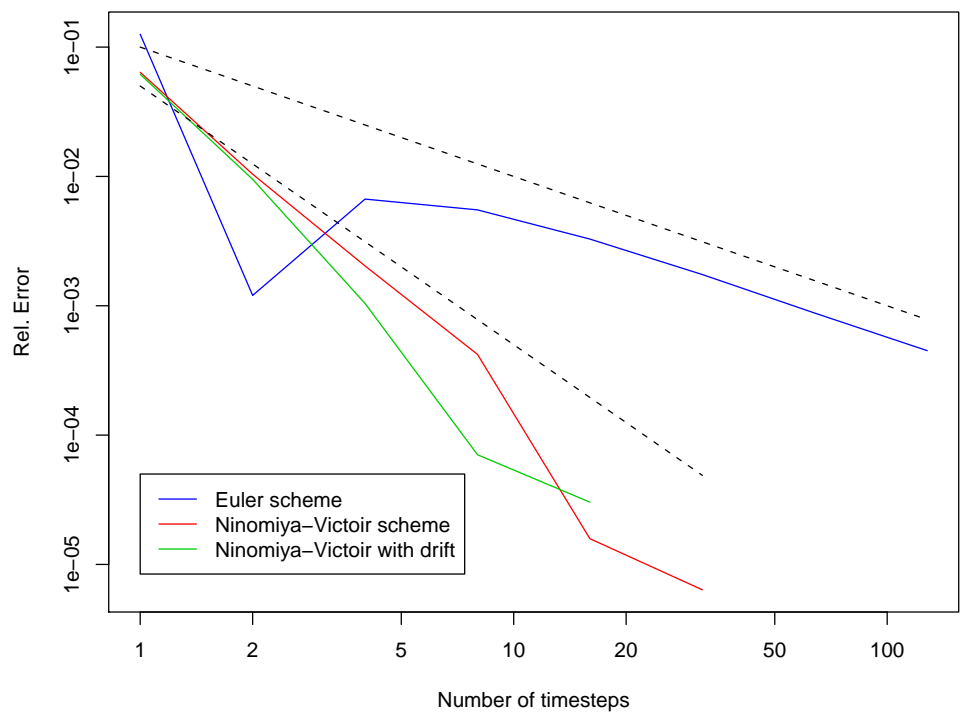

Fig. 4 Order of convergence for the generalized SABR model. Figure from [2].

\begin{tabular}{|l|c|c|c|c|}
\hline Method & $K$ & $M$ & Rel. Error & Time \\
\hline Euler & 32 & 8192000 & 0.00174 & $91.94 \mathrm{sec}$ \\
\hline Ninomiya-Victoir & 4 & 2048000 & 0.00204 & $13.93 \mathrm{sec}$ \\
\hline NV with drift & 4 & 1024000 & 0.00104 & $2.88 \mathrm{sec}$ \\
\hline
\end{tabular}

Table 1 Computational time for the generalized SABR model

such that the weak error is of order $10^{-3}$. We see that the computational time needed for the adjusted splitting method (40) is indeed considerably smaller than the time for the classical one (31). The other numerical parameter (the number $M$ of samples for the quasi Monte Carlo integration, restricted to be a power of 2) was chosen such that the integration error (i.e., the error in the computation of the expected value) is of order $10^{-5}$. Indeed, we focus on the discretization problem here, an we do not want our results to be overshadowed by the integration error. Note that in the case of the Euler scheme, one has to compute a 64-dimensional integral, whereas in the case of the Ninomiya-Victoir scheme (with or without drift), the integration only needs to be performed on an eight-dimensional space. This explains why the $M$ can be chosen smaller for the Ninomiya-Victoir splitting as compared to the Euler scheme, as quasi Monte Carlo is known to work better when the dimension is smaller despite not suffering from the curse of dimensionality.

In [2] similar results were reported for the multi-asset case. More precisely, the authors of [2] also applied it to the case of four assets, meaning an eight-dimensional model. But, in fact, the stochastic splitting method can be used in even higher dimensions. For instance, we used it in [4] in order to obtain reference solutions for options depending on up to 100 assets for a pure local volatility model, coupled with 
quasi Monte Carlo or Monte Carlo methods for the integration step. In that case, it is difficult if not impossible to obtain reliable reference values, but the method seems to perform very well.

\section{Calibration of the double mean reverting model}

The double mean reverting model goes back to Jim Gatheral [33]. Its main advantage is that it allows joint calibration to market data for option prices on an index like the S \& P 500 index (SPX) and a corresponding implied volatility index (like the VIX). The model is given by

$$
\begin{aligned}
& d S_{t}=\sqrt{v_{t}} S_{t} d W_{t}^{1}, \\
& d v_{t}=\kappa_{1}\left(v_{t}^{\prime}-v_{t}\right) d t+\xi_{1} v_{t}^{\alpha_{1}} d W_{t}^{2}, \\
& d v_{t}^{\prime}=\kappa_{2}\left(\theta-v_{t}^{\prime}\right) d t+\xi_{2} v_{t}^{\prime \alpha_{2}} d W_{t}^{3},
\end{aligned}
$$

where the Brownian motions $W_{i}$ are all correlated with $E\left[d W_{t}^{i} d W_{t}^{j}\right]=\rho_{i j} d t$. Again, it is natural to interpret $v_{t}$ as the (stochastic) volatility of the (discounted) asset price process $S_{t}$ - or rather, as the stochastic variance. Conforming to stylized facts about the volatility, $v_{t}$ is a mean-reverting process due to the form of the drift, but unlike traditional stochastic volatility models, the long-term mean $v_{t}^{\prime}$ is itself a (meanreverting) stochastic process, hence the name "double mean reverting" model.

Typically, one of the most numerically challenging tasks in financial engineering is the calibration of a model such as (41), i.e., the fitting of the model parameters $\left(\kappa_{1}, \kappa_{2}, \xi_{1}, \xi_{2}, \alpha_{1}, \alpha_{2}, \rho_{12}, \rho_{13}, \rho_{23}\right.$, but also $v_{0}$ and $v_{0}^{\prime}$ which, unlike $S_{0}$, are not directly observable) to market data, in particular to market option prices. Indeed, even though the model itself assumes these parameters to be constant, in reality they typically change frequently, which means that the model has to be re-calibrated on a regular bases, say daily.

In the case of the double mean reverting model, practical experience seems to show that $\theta, \kappa_{1}, \kappa_{2}, \rho_{23} \alpha_{1}$ and $\alpha_{2}$ are fairly constant in time, implying that they can be excluded from the daily re-calibration. In fact, [3] found that the data available did not suffice to successfully estimate $\alpha_{2}$. Hence, it was assumed to have the same value as $\alpha_{1}$, which was calibrated to $\alpha_{1}=0.94$. Hence, for the purpose of their numerical study, [3] assumed $\theta, \kappa_{1}, \kappa_{2}, \rho_{23} \alpha_{1}$ and $\alpha_{2}$ to be given (by parameters which where themselves, of course, calibrated to the market data) - leaving us with the task of fast calibration of the parameters $\xi_{1}, \xi_{2}, \rho_{12}$ and $\rho_{13}$. In the context of [3], "market data" mean the prices of vanilla (i.e., European put and call options) on SPX and on VIX. The general calibration procedure proposed was the following:

1. Given a time series of VIX data, linear regression allows to construct time series for the processes $v_{t}$ and $v_{t}^{\prime}$. Out of these, a least-square optimization is used to estimate $\theta, \kappa_{1}$ and $\kappa_{2}$. Moreover, the correlation between $v_{t}$ and $v_{t}^{\prime}$ gives $\rho_{23}$. A similar regression on VIX time series data gives an estimate for $\alpha_{1}$. 
2. Note that options on VIX depend only on $v_{t}$ and $v_{t}^{\prime}$ now, but not on $S_{t}$. Hence, one can calibrate $\xi_{1}$ and $\xi_{2}$ directly to VIX options, without needing to simulate the $S_{t}$ component, i.e., without adding any constraints to $\rho_{12}$ and $\rho_{13}$. The calibration boils down to a least-squares minimization of misfits of VIX-option prices from the model to the quoted market prices. The minimization was done using a Levenberg-Marquardt algorithm, for the option pricing algorithm the authors of [3] tested the Euler scheme and a variant of the Ninomiya-Victoir scheme for the discretization of the SDE and both classical and quasi Monte Carlo for the computation of the expected value.

3. Having obtained $\xi_{1}$ and $\xi_{2}$ from the previous step, they used options on the SPX to calibrate the remaining parameters $\rho_{12}$ and $\rho_{13}$. The procedure is very similar to the calibration of $\xi_{1}$ and $\xi_{2}$, except that now the full three-dimensional SDE needs to be solved.

In [3] the calibration was done for two particular days, namely April 3, 2007 (before the financial crisis) and September 15, 2011 (after the financial crisis). The fits to SPX options are quite good, especially for maturities which are not too small. The fit to VIX options is slightly worse, but in that time VIX options were also less liquid than today. Regarding the numerical algorithms, the Ninomiya-Victoir splitting method (with an additional splitting in the drift, not unlike the one presented in [2]) performs much better for the calculation of VIX options, where the classical Euler method would requires 500 time-steps as compared to 30 time-steps for the splitting method in order to achieve the required accuracy. Thereby, for this example, the Ninomiya-Victoir scheme reduces the calibration time for the VIX-step by a factor of around five. For the SPX options, the Euler scheme surprisingly gave sufficiently accurate results already for 30 time-steps, which implies that for this case the Euler scheme turned out to be seemingly preferable to the Ninomiya-Victoir scheme, taking costs into account. Note, however, that the error plots in Figure 5 reveal that this conclusion is deceptive, as the weak approximation error changes its sign around the critical number of 30 time-steps. Even though this effect was persistent in both for both market data (and the accordingly calibrated parameters), the picture might change in other regime, leading to added benefits for applying the Ninomiya-Victoir splitting scheme also for the SPX-data calibration step. In total, the authors of [3] report that their implementation can do the re-calibration to market data in about 5 seconds using the Ninomiya-Victoir splitting scheme.

\section{Calibration of the Heath-Jarrow-Morton model}

Finally, we want to present an application of the Ninomiya-Victoir weak splitting method to a true SPDE given by Dörsek and Teichmann [23], namely the fast calibration of a general, infinite-dimensional Heath-Jarrow-Morton model for interest rate dynamics, see [39]. We should also note alternative numerical treatments of the full, infinite dimensional HJM model in [9] and [57]. 

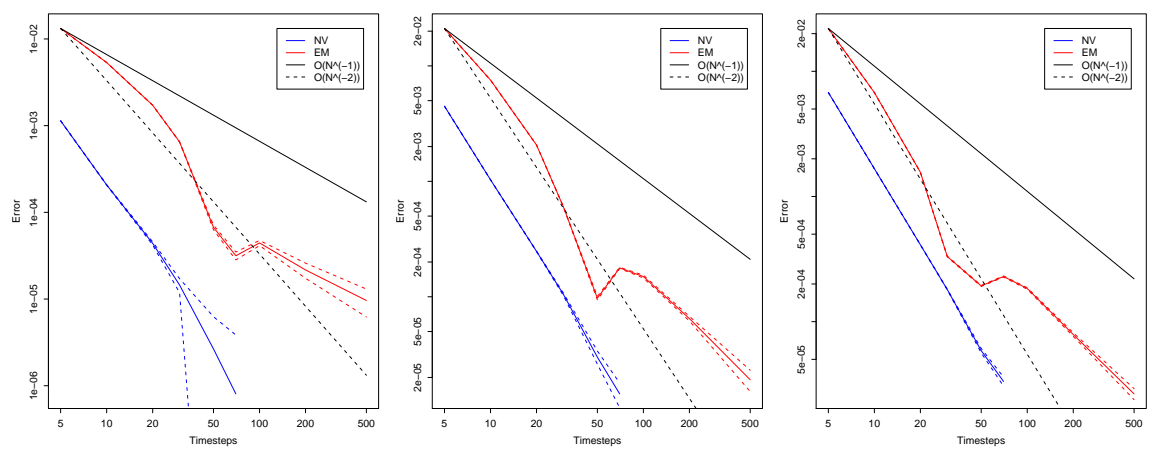

Fig. 5 Errors for the Euler and the Ninomiya Victoir schemes for three different SPX options. (Data from September 15, 2011, dotted lines indicate Monte Carlo confidence intervals. Figure taken from [3].)

We start with a short description of the model. Let $P(t, T)$ denote the price of a zero-coupon bond with maturity $T$ at time $t$. Of course, this entity only makes sense if $t \leq T$. The (instantaneous) forward rate at time $t$ for the maturity $T$ is defined by

$$
f(t, T)=-\frac{\partial}{\partial T} \log P(t, T),
$$

implying the natural inverse relation $P(t, T)=\exp \left(-\int_{t}^{T} f(t, u) d u\right)$, which explains why we call $f$ an interest rate. Unlike many other models, in which only the short rate $f(t, t)$ or only the rates $f\left(t, T_{1}\right), \ldots, f\left(t, T_{n}\right)$ for finitely many maturities are modeled, [39] propose an infinite-dimensional model for the whole forward rate curve $(f(t, T))_{T \in[t, \infty[}$. In order to avoid working with time-dependent state spaces, we introduce the parametrization $r_{t}(x)=f(t, t+x)$ in time to maturity $x=T-t \geq 0$. Then the HJM model corresponds to the SPDE

$$
d r_{t}=\left(\frac{\partial}{\partial x} r_{t}+\alpha_{H J M}\left(r_{t}\right)\right) d t+\sum_{i=1}^{d} \sigma_{i}\left(r_{t}\right) d B_{t}^{i}
$$

Here, we restrict ourselves to a finite number $d$ of driving Brownian motions, which can be justified empirically, but is not strictly necessary for the HJM model. Moreover, we note that there are inherent restrictions on the vector fields imposed by no-arbitrage constraints, which boil down to the relation

$$
\alpha_{H J M}(h)(x)=\sum_{i=1}^{d} \sigma_{i}(h)(x) \int_{0}^{x} \sigma_{i}(h)(y) d y,
$$

where $x \geq 0$ and $h$ takes values in the state space $H$, a suitably weighted Sobolev space, see [23] for details on $H$ and on further regularity requirements on $\sigma_{i}$. 
Next, we describe the splitting. Note that the diffusion vector fields do not pose any additional complications as compared to the finite dimensional case, as they are (assumed to be) continuous vector fields on $H$. This is evidently not the case for the Stratonovich drift vector field $\sigma_{0}(h)=\frac{\partial}{\partial x} h+\alpha_{H J M}(h)-\frac{1}{2} D \sigma_{i}(h) \cdot \sigma_{i}(h)$, where the unbounded operator $\frac{\partial}{\partial x}$ appears. (Recall that the solution $r_{t}$ cannot, in fact, be written in Stratonovich form.) Hence, they additionally split $\sigma_{0}=\sigma_{0,1}+\sigma_{0,2}$ with $\sigma_{0,1}=\frac{\partial}{\partial x}$ and $\sigma_{0,2}=\alpha_{H J M}-\frac{1}{2} D \sigma_{i} \cdot \sigma_{i}$. Here we note that the flow of $\sigma_{0,1}$ is obviously given by the shift operator $S_{t}(h)(x)=h(x+t)$, so that the $e^{s \sigma_{0,1}}=S_{s}$ is given in closed form. Regarding the diffusion vector fields, the authors suggest to use the parametric form

$$
\sigma_{j}(h, v)(x)=\left(\alpha_{j, 0}+\alpha_{j, 1} x\right) e^{-\beta x} \tanh \left(c_{j} e^{v} \int_{0}^{t_{j}} h(s) d s\right)
$$

which includes a stochastic volatility component $v$, and $\alpha_{j, i}, \beta, c_{j}$ and $t_{j}$ are parameters, which need to be estimated. Moreover, they choose $d=3$.

The authors of [23] calibrate against 120 market prices of caplets, again using a Levenberg-Marquardt type algorithm for the optimization. In total, they report that it takes about half a second to compute these 120 option prices to the required accuracy (on a workstation with 16 cores), and the total calibration can be done in 14.5 minutes.

Acknowledgements Harald Oberhauser is grateful for the support of the ERC (grant agreement No.291244 Esig) and the Oxford-Man Institute of Quantitative finance.

\section{References}

1. Bain, A., Crişan, D.: Fundamentals of Stochastic Filtering. Springer, New York, NY (2009)

2. Bayer, C., Friz, P., Loeffen, R.: Semi-closed form cubature and applications to financial diffusion models. Quant. Finance 13(5), 769-782 (2013)

3. Bayer, C., Gatheral, J., Karlsmark, M.: Fast Ninomiya-Victoir calibration of the double-meanreverting model. Quantitative Finance 13(11), 1813-1829 (2013)

4. Bayer, C., Laurence, P.: Asymptotics beats Monte Carlo: The case of correlated CEV baskets. Comm. Pure Appl. Math. 67(10), 1618-1657 (2014)

5. Bayer, C., Teichmann, J.: Cubature on Wiener space in infinite dimension. Proc. R. Soc. Lond. Ser. A Math. Phys. Eng. Sci. 464(2097), 2493-2516 (2008)

6. Bensoussan, A., Glowinski, R.: Approximation of Zakai equation by the splitting up method. In: Stochastic Systems and Optimization, Lecture Notes in Control and Inform. Sci., vol. 136, pp. 257-265. Springer, Berlin (1989)

7. Bensoussan, A., Glowinski, R., Răşcanu, A.: Approximation of the Zakai equation by the splitting up method. SIAM J. Control Optim. 28(6), 1420-1431 (1990)

8. Bensoussan, A., Glowinski, R., Răşcanu, A.: Approximation of some stochastic differential equations by the splitting up method. Appl. Math. Optim. 25(1), 81-106 (1992)

9. Björk, T., Szepessy, A., Tempone, R., Zouraris, G.E.: Monte Carlo Euler approximations of HJM term structure financial models. BIT 53(2), 341-383 (2013)

10. Brigo, D., Hanzon, B.: On some filtering problems arising in mathematical finance. Insurance: Mathematics and Economics 22(1), 53-64 (1998) 
11. Brockett, R.W.: Volterra series and geometric control theory. Automatica 12(2), 167 - 176 (1976)

12. Caruana, M., Friz, P., Oberhauser, H.: A (rough) pathwise approach to a class of non-linear stochastic partial differential equations. Ann. Inst. H. Poincaré Anal. Non Linéaire 28(1), 27-46 (2011)

13. Clark, J.: The design of robust approximations to stochastic differential equations in nonlinear filtering. In: J. Skwirzynsky (ed.) Communication Systems in Random Processes Theory, pp. 721-734. Sijthoff,Nordhoff (1978)

14. Clark, J., Crisan, D.: On a robust version of the integral representation formula of nonlinear filtering. Probability Theory and Related Fields 133(1), 43-56 (2005)

15. Crandall, M.G., Ishii, H., Lions, P.L.: User's guide to viscosity solutions of second order partial differential equations. Bull. Amer. Math. Soc. (N.S.) 27(1), 1-67 (1992)

16. Crisan, D., Diehl, J., Friz, P., Oberhauser, H.: Robust filtering: multdimensional noise and multidimensional observation. Annals of Applied Probability 23(5), 2139-2160 (2013)

17. Crisan, D., Rozovskii, B.: The Oxford Handbook of Nonlinear Filtering. Oxford Handbooks. Oxford University Press (2011)

18. Csomós, P., Faragó, I., Havasi, Á.: Weighted sequential splittings and their analysis. Comput. Math. Appl. 50(7), 1017-1031 (2005)

19. Da Prato, G., Zabczyk, J.: Stochastic Equations in Infinite Dimensions, 2nd edn. Cambridge University Press, Cambridge (2014)

20. Debussche, A.: Weak approximation of stochastic partial differential equations: the nonlinear case. Math. Comp. 80(273), 89-117 (2011)

21. Diehl, J., Friz, P., Gassiat, P.: Stochastic control with rough paths. arXiv preprint arXiv:1303.7160 (2013)

22. Dörsek, P., Teichmann, J.: A semi-group point of view on splitting schemes for stochastic (partial) differential equations. arXiv preprint arXiv:1011.2651 (2010)

23. Dörsek, P., Teichmann, J.: Efficient simulation and calibration of general HJM models by splitting schemes. SIAM J. Financial Math. 4(1), 575-598 (2013)

24. Elliott, R.J., Glowinski, R.: Approximations to solutions of the Zakai filtering equation. Stochastic Analysis and Applications 7(2), 145-168 (1989)

25. Fleming, W.H., Soner, H.M.: Controlled Markov Processes and Viscosity Solutions, Stochastic Modelling and Applied Probability, vol. 25. Springer, New York (2006)

26. Fliess, M.: Fonctionnelles causales non linéaires et indéterminées non commutatives. Bull. Soc. Math. France 109(1), 3-40 (1981)

27. Florchinger, P., Le Gland, F.: Time-discretization of the Zakai equation for diffusion processes observed in correlated noise. Stochastics and Stochastic Reports 35(4), 233-256 (1991)

28. Frey, R., Runggaldier, W.J.: A nonlinear filtering approach to volatility estimation with a view towards high frequency data. International Journal of Theoretical and Applied Finance 4(2), 199-210 (2001)

29. Friz, P., Hairer, M.: A Course on Rough Paths: With an Introduction to Regularity Structures. Universitext. Springer International Publishing (2014)

30. Friz, P., Oberhauser, H.: On the splitting-up method for rough (partial) differential equations. Journal of Differential Equations 251(2), 316 - 338 (2011)

31. Friz, P., Oberhauser, H.: Rough path stability of (semi-)linear SPDEs. Probability Theory and Related Fields 158(1), 401-434 (2014)

32. Friz, P.K., Victoir, N.B.: Multidimensional Stochastic Processes as Rough Paths: Theory and Applications. Cambridge University Press, Cambridge (2010)

33. Gatheral, J.: Consistent modeling of SPX and VIX options. In: Proceedings of the Fifth World Congress of the Bachelier Finance Society. London (2008)

34. Gençay, R., Selçuk, F., Whitcher, B.J.: An Introduction to Wavelets and Other Filtering Methods in Finance and Economics. Academic Press, San Diego, CA (2002)

35. Gubinelli, M.: Controlling rough paths. J. Funct. Anal. 216(1), 86-140 (2004)

36. Gyöngy, I., Krylov, N.: On the splitting-up method and stochastic partial differential equations. Ann. Probab. 31(2), 564-591 (2003) 
37. Gyöngy, I., Krylov, N.: Accelerated numerical schemes for PDEs and SPDEs. In: D. Crisan (ed.) Stochastic Analysis 2010, pp. 131-168. Springer, Berlin Heidelberg (2011)

38. Hausenblas, E.: Approximation for semilinear stochastic evolution equations. Potential Anal. 18(2), 141-186 (2003)

39. Heath, D., Jarrow, R., Morton, A.: Bond pricing and the term structure of interest rates: A new methodology for contingent claims valuation. Econometrica 60(1), 77-105 (1992)

40. Hida, T.: Brownian Motion. Springer, New York, NY (1980)

41. Ikeda, N., Watanabe, S.: Stochastic Differential Equations and Diffusion Processes, 2nd edn. North-Holland, Amsterdam (1989)

42. Jentzen, A., Kloeden, P.E.: The numerical approximation of stochastic partial differential equations. Milan J. Math. 77, 205-244 (2009)

43. Karatzas, I., Shreve, S.E.: Brownian Motion and Stochastic Calculus, 2nd edn. Springer, New York, NY (1991)

44. Kloeden, P.E., Platen, E.: Numerical Solution of Stochastic Differential Equations. Springer, Berlin Heidelberg (1992)

45. Kohatsu-Higa, A., Tankov, P.: Jump-adapted discretization schemes for Lévy-driven SDEs. Stochastic Process. Appl. 120(11), 2258-2285 (2010)

46. Kusuoka, S.: Approximation of expectation of diffusion processes based on Lie algebra and Malliavin calculus. In: Advances in Mathematical Economics, vol. 6, pp. 69-83. Springer (2004)

47. Le Gland, F.: Splitting-up approximation for SPDEs and SDEs with application to nonlinear filtering. In: Stochastic Partial Differential Equations and Their Applications, pp. 177-187. Springer, Berlin Heidelberg (1992)

48. Lototsky, S., Mikulevicius, R., Rozovskii, B.L.: Nonlinear filtering revisited: a spectral approach. SIAM J. Control Optim. 35(2), 435-461 (1997)

49. Lyons, T.: Differential equations driven by rough signals. I. An extension of an inequality of L. C. Young. Math. Res. Lett. 1(4), 451-464 (1994)

50. Lyons, T.: Differential equations driven by rough signals. Rev. Mat. Iberoamericana 14(2), 215-310 (1998)

51. Lyons, T.: Systems controlled by rough paths. In: European Congress of Mathematics, pp. 269-281. Eur. Math. Soc., Zürich (2005)

52. Lyons, T., Qian, Z.: System Control and Rough Paths. Oxford University Press, Oxford (2002)

53. Lyons, T., Victoir, N.: Cubature on Wiener space. Proc. R. Soc. Lond. Ser. A Math. Phys. Eng. Sci. 460(2041), 169-198 (2004)

54. Lyons, T.J., Caruana, M., Lévy, T.: Differential Equations Driven by Rough Paths, Lecture Notes in Mathematics, vol. 1908. Springer, Berlin (2007)

55. Mandelbrot, B.B., Van Ness, J.W.: Fractional Brownian motions, fractional noises and applications. SIAM Review 10(4), 422-437 (1968)

56. Nagase, N.: Remarks on nonlinear stochastic partial differential equations: an application of the splitting-up method. SIAM J. Control Optim. 33(6), 1716-1730 (1995)

57. Ninomiya, M.: Application of the Kusuoka approximation with a tree-based branching algorithm to the pricing of interest-rate derivatives under the HJM model. LMS J. Comput. Math. 13, 208-221 (2010)

58. Ninomiya, S., Victoir, N.: Weak approximation of stochastic differential equations and application to derivative pricing. Appl. Math. Finance 15(1-2), 107-121 (2008)

59. Øksendal, B.: Stochastic Differential Equations: An Introduction with Applications. Springer, Berlin (2003)

60. Protter, P.E.: Stochastic Integration and Differential Equations: A New Approach. Springer, Berlin Heidelberg (1992)

61. Protter, P.E.: Stochastic Integration and Differential Equations. Springer, Berlin Heidelberg (2005)

62. Răşcanu, A., Tudor, C.: Approximation of stochastic equations by the splitting up method. In: Qualitative Problems for Differential Equations and Control Theory, pp. 277-287. World Sci. Publ., River Edge, NJ (1995) 
63. Revuz, D., Yor, M.: Continuous Martingales and Brownian Motion, 3rd edn. Springer, Berlin (1999)

64. Schwab, C., Gittelson, C.J.: Sparse tensor discretizations of high-dimensional parametric and stochastic PDEs. Acta Numerica 20, 291-467 (2011)

65. Sun, M., Glowinski, R.: Pathwise approximation and simulation for the Zakai filtering equation through operator splitting. Calcolo 30(3), 219-239 (1993)

66. Sussmann, H.J.: Semigroup representations, bilinear approximation of input-output maps, and generalized inputs. In: M. Beckmann, H.P. Künzi, G. Marchesini, S.K. Mitter (eds.) Mathematical Systems Theory, vol. 131, pp. 172-191. Springer, Berlin Heidelberg (1976)

67. Teichmann, J.: Another approach to some rough and stochastic partial differential equations. Stochastics and Dynamics 11(2-3), 535-550 (2011)

68. Yan, Y.: Galerkin finite element methods for stochastic parabolic partial differential equations. SIAM J. Numer. Anal. 43(4), 1363-1384 (2005) 